Труды Москов. Матем. Общ. Tom $71(2010)$
Trans. Moscow Math. Soc. 2010, Pages 175-207 S 0077-1554(2010)00184-7

Article electronically published on December 21, 2010

\title{
CANARD CYCLES IN GENERIC FAST-SLOW SYSTEMS ON THE TORUS
}

\author{
I. V. SHCHUROV
}

\begin{abstract}
In generic fast-slow systems with a single parameter on the two-dimensional torus, for arbitrarily small values of this parameter there exist attracting canard cycles. This is a key distinction between the dynamics on the torus and the dynamics of similar systems on the plane. This has already been proved for systems with a convex slow curve. This paper looks at systems with a nonconvex slow curve. Upper and lower estimates for the number of canard cycles are obtained. An open set of systems having a preassigned number of attracting canard cycles is constructed.
\end{abstract}

\section{INTRODUCTION}

We consider a fast-slow system on the torus:

$$
\left\{\begin{array}{l}
\dot{x}=f(x, y, \varepsilon), \\
\dot{y}=\varepsilon g(x, y, \varepsilon),
\end{array} \quad(x, y) \in \mathbb{T}^{2}, \quad \varepsilon \in(\mathbb{R}, 0) .\right.
$$

In such systems a new effect appears, which is not observed in analogous systems on the plane. This effect is related to attracting closed solutions that are canards 1, 2, which travel a distance near the unstable part of the slow surface that is bounded below as $\varepsilon \rightarrow 0$.

This effect was first described by Ilyashenko and Guckenheimer [3]. It was shown that for a certain concrete family of systems with no additional parameters there exists a sequence of intervals accumulating to zero such that, for every $\varepsilon$ in these intervals, the system has a pair of canard cycles, one attracting and the other repelling, and this pair is unique. This is a key distinction between the dynamics on the torus and the dynamics on the plane, where canard solutions of this type are observed in the presence of an additional parameter (see, for example, [4]).

Subsequently this assertion was generalized to the case of an arbitrary fast-slow system on the torus that has a convex slow curve and satisfies certain local conditions of a generic nature (see [5]).

This paper extends the studies described above and is devoted to considering the case of a nonconvex slow curve. It turns out that, depending on the form of the slow curve and the values of the derivative $f_{x}^{\prime}$ on it, for an arbitrarily small value of the parameter several pairs of stable and unstable canard cycles can be observed. There are no more of them than the number of folds of the slow curve under the projection along the direction of fast motion. At the same time, for any preassigned number $k$ it is possible to construct an open set of systems that have $k$ pairs of canard cycles for an arbitrarily small value of the parameter $\varepsilon$.

2010 Mathematics Subject Classification. Primary 34E17; Secondary 34E15, 37G15, 70K70.

Key words and phrases. Fast-slow system, attracting canard cycle, slow curve, dynamical system.

This research was partially supported by the Russian Foundation for Basic Research (grant no. 1001-00739-a). 


\section{MAIN RESULTS}

In this section we present the definitions we need and state the main results of the paper.

2.1. The preliminary statement of the main result. In this subsection we state two results we obtain in this paper rigorously, whilst minimizing the number of preliminary constructions. In Subsections 2.2 and 2.3 we state similar but more precise results.

We consider a fast-slow system on the torus:

$$
\left\{\begin{array}{l}
\dot{x}=f(x, y, \varepsilon), \\
\dot{y}=\varepsilon g(x, y, \varepsilon),
\end{array} \quad(x, y) \in \mathbb{T}^{2} \cong \mathbb{R}^{2} /\left(2 \pi \mathbb{Z}^{2}\right), \varepsilon \in(\mathbb{R}, 0)\right.
$$

where the functions $f$ and $g$ are smooth enough that all our subsequent statements hold. Throughout the paper we assume that the speed of the slow motion is separated from zero (for definiteness, $g>0$ ) and the lift of the slow curve $M:=\{(x, y) \mid f(x, y, 0)=0\}$ to the covering coordinate plane lies in the interior of the square $\{|x|<\pi,|y|<\pi\}$. We also require that $f_{x}^{\prime}(x, y, 0) \neq 0$ for any point $(x, y) \in M$ other than the fold points. These conditions define an open set in the space of all fast-slow systems on the torus. Without loss of generality we can assume that $f(x, 0,0)>0$ (outside the interior of the slow surface the fast motion is directed in the positive direction). Since we are only interested in the phase curves of the system (2.1) and not in their parametrization, we can divide the vector field of the system by $g$ and assume without loss of generality that $g \equiv 1$.

We are interested in the canard solutions, that is, solutions that, as $\varepsilon \rightarrow 0^{+}$, travel a distance separated from zero near the unstable part of the slow curve. The precise definition will be given below (see Definition 2.4 in the next subsection). Our main goal is to analyse canard cycles that perform one revolution along the $y$-axis.

Theorem 2.1 (upper estimate for the number of canard cycles). A generic system of the form (2.1) has the following property. On the $\varepsilon$-axis there exists a sequence of intervals accumulating to zero such that for every $\varepsilon$ that belongs to one of these intervals the system has attracting closed canard solutions (canard cycles) performing one revolution along the $y$-axis. The basin of attraction of each of these cycles (on the entire torus) has a measure that is uniformly bounded below. There are no more than half as many of these cycles as the number of jump points (that is, points on the curve $M$ at which the tangent to $M$ is vertical).

Theorem 2.1 is a corollary of Theorem 2.5. which is more precise and stronger. We state it in the next subsection, together with Proposition 2.6. It gives an upper bound for the number of canard cycles a generic system can have. When $M$ is a convex curve (more precisely, contains exactly two jump points), the theorem was proved in [5]. In this paper we consider the case of a nonconvex slow curve $M$.

Theorem 2.2 (sharp estimate). There exists an open set of systems for which the upper bound for the number of canard cycles given in Theorem 2.1 is attained: the number of canard cycles is equal to the number of folds on the slow curve.

Theorem 2.2 is proved in 9 . In $\$ 2.3$ a special result of this type is stated in the simplest nonconvex case: we give explicit necessary and sufficient conditions for the maximal number of attracting canard cycles to exist in this case; as a result we describe the open set of systems appearing in this theorem explicitly. 
2.2. The general case: an upper bound for the number of canard cycles. We need to impose additional requirements on the system (2.1): this is to exclude from consideration systems satisfying finitely many conditions consisting of a type of equation which will be formulated later on in the paper. In particular, throughout what follows we require that the following additional nondegeneracy condition holds: all the fold points of the slow curve $M$ are nondegenerate, that is,

$$
\left.\frac{\partial^{2} f(x, y, 0)}{\partial x^{2}}\right|_{G} \neq 0,\left.\quad \frac{\partial f(x, y, 0)}{\partial y}\right|_{G} \neq 0
$$

for every fold point $G$ of the curve $M$. We also require that all the fold points have different $y$-coordinates:

$$
y\left(G_{1}\right) \neq y\left(G_{2}\right) .
$$

In the course of proving the results we shall need to impose two more nondegeneracy conditions of the same type on the system; to state them we need additional constructions (see (5.4) and (5.17)).

Definition 2.3. A fold point $G$ of the slow curve $M$ is called a forward (reverse) jump point if in a small neighbourhood of it the part of the slow curve is situated on the left (on the right) of $G$.

We denote by $G^{ \pm}$the left-most $(+)$and the right-most $(-)$jump points (they are defined uniquely due to the nondegeneracy conditions (2.2) and (2.3)).

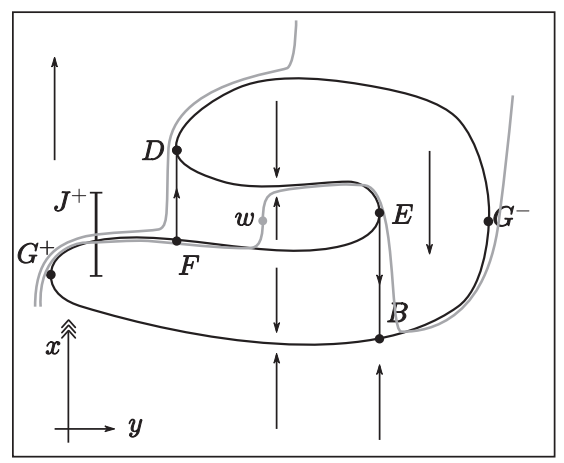

FiguRE 1. The simplest nonconvex case: 4 jump points; $E$ and $G^{-}$are forward jump points; $G^{+}$and $D$ are reverse jump points. The triple arrow shows the direction of fast motion.

Let $J^{+}$be some fixed segment that is a transversal to the unstable part of the slow curve $M$, is close to the reverse jump point $G^{+}$(see Figure 1), and does not intersect the stable part of the slow curve. Its exact location will be defined below (see p.182).

Definition 2.4. A trajectory is called canard if it intersects $J^{+}$.

The trajectory starting from $J^{+}$in reverse time is rapidly attracted to the slow curve and covers almost all the distance to the jump point near the unstable part of the slow curve. Thus, the formal Definition 2.4 corresponds to the heuristic description of canard solutions given in $\$ 2.1$.

Figure 1 depicts the simplest case of a nonconvex slow curve. Consider the trajectory passing near the repelling arc of the slow curve $D G^{-}$. Reverse the time: then the arc $D G^{-}$ becomes attracting, and the trajectory moves to the left near this arc right up to the reverse jump near $D$, after which it is attracted to the part $G^{+} F$ again moving to the left 
right up to the jump point $G^{+}$. Consequently, it intersects the segment $J^{+}$and thus is canard in the sense of the definition given above. In more complicated cases, a trajectory passing near some unstable arc of the slow curve in reverse time may undergo a series of reverse jumps, after which it intersects $J^{+}$.

We consider a nondegenerate system of the form (2.1) with $2 N$ jump points.

Theorem 2.5. There exists a positive number $k \leq N$, a sequence of disjoint segments $R_{n}:=\left[\alpha_{n}, \beta_{n}\right]$, and a set of $k$ sequences of disjoint nonempty intervals $C_{n}^{i} \subset R_{n}, i=$ $1, \ldots, k$, such that the following properties hold (see Figure 2).

1. $\left|R_{n}\right|=O\left(e^{-C n}\right)$.

2. $\alpha_{n}=O(1 / n)$.

3. For any small $\varepsilon$ that does not belong to the union of the $R_{n}$, the system has exactly two hyperbolic periodic trajectories, stable and unstable, and the unstable one is canard.

4. For any small $\varepsilon$ in $C_{n}^{i}$ the system has exactly $i$ pairs of hyperbolic periodic trajectories (stable and unstable), and all the periodic trajectories are canard.

5. For any small $\varepsilon>0$ the total number of limit cycles that perform 1 revolution along the $y$-axis does not exceed $2 k$.

Proposition 2.6. For every $\varepsilon \in C_{n}^{i}$ the measure of the basin of attraction (repulsion) of every canard cycle is uniformly separated from zero.

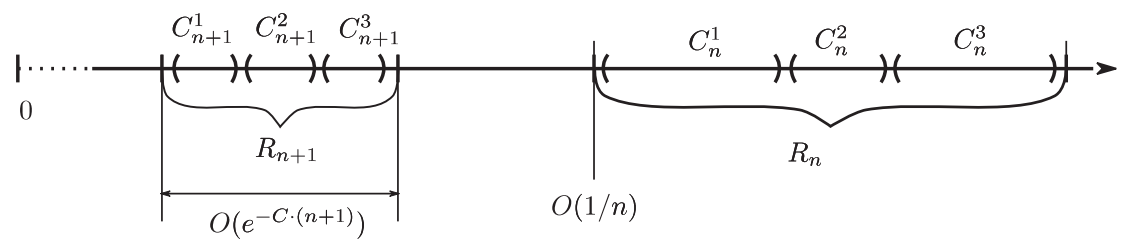

Figure 2. Domains on the ray $\varepsilon>0$.

The number $k$ depends on the shape of the slow curve $M$ and the values of the integrals of the function $f_{x}^{\prime}$ over special sets of arcs of the slow curve and can be calculated explicitly from the system.

2.3. A lower estimate for the number of canard cycles. We consider a system of the form (2.1) that has four folds: two of them are forward jump points, and two reverse jump points. By Theorem 2.5 this system has at most two pairs of canard cycles. In this subsection we state explicit conditions under which the system has exactly two pairs of canard cycles (or equivalently, two attracting canard cycles).

We consider the case depicted in Figure 1. The other possible mutual positions of the jump points can be considered in a similar fashion.

We introduce the notation

$$
\Phi(U)=\left.\int_{U} f_{x}^{\prime}\right|_{(x(y), y, 0)} d y,
$$

where $U$ is some arc of the slow curve $M$ and $x=x(y)$ defines the graph of this arc. By emitting two close trajectories from a neighbourhood of the beginning of this arc and integrating the variational equation for them, it is easy to see that this integral defines the rate of 'convergence' (or 'scattering') of the trajectories over the time of motion near the arc. This integral is negative if $U$ is an attracting arc, and positive if $U$ repels. 
Theorem 2.7. Suppose that a nondegenerate system of the form (2.1) corresponds to Figure 1 and the following additional relations hold, called 'balancing conditions':

1. $\Phi\left(G^{+} E\right)+\Phi\left(B G^{-}\right)>0$.

2. $\Phi\left(G^{+} F\right)+\Phi(D E)+\Phi\left(B G^{-}\right)<0$.

Then in Theorem 2.5 the number $k$ of pairs of canard cycles is maximal and given by $N=2$.

\section{The Poincaré map: OVERVIEW of the PRoOF}

3.1. A panoramic view of the proof. In this subsection we present, at a descriptive level, the arguments we use to prove the main result and give references to the corresponding places in the paper.

We shall follow the main ideas of $\underline{3}$. The key tool in the subsequent analysis is the Poincaré map $P_{\varepsilon}$ from the transversal $\Gamma:=\{y=-\pi\}$ to itself along the phase curves (2.1). Since $g \equiv 1$ this map is a diffeomorphism of the circle and defined everywhere. Its periodic (and in particular, fixed) points correspond to periodic trajectories of the system. We denote the graph of the map $P_{\varepsilon}$ by $\gamma_{\varepsilon}$. The fixed points of the Poincaré map correspond to the intersection points of the graph with the diagonal $\mathcal{D}:=\{y=x\}$.

In practice, at almost all the points of the preimage of the circle (with the exception of a small arc), the map $P_{\varepsilon}$ has a derivative that is close to 0 , that is, it is strongly contracting. At the same time, the remaining small arc is strongly expanded under the action of $P_{\varepsilon}$ and its image is almost the whole circle; at the points of this arc the derivative of the Poincaré map can be very large.

Geometrically this corresponds to the graph $\gamma_{\varepsilon}$ being contained in the union of two thin narrow strips: vertical and horizontal; see Figure 5 . The width of the strips is exponentially small together with $\varepsilon$ (Lemma 3.1). The strips are constructed in such a way that all the points of the graph contained in the vertical strip correspond to canard solutions of the system.

Outside the rectangle that is the intersection of the strips, the slope of the graph is either very small (the graph is almost horizontal) or very large (the graph is almost vertical). Thus points of the graph $\gamma_{\varepsilon}$ at which the derivative of $P_{\varepsilon}$ is equal to 1 (we call them neutral) can only be located inside that rectangle. The neutral points divide the graph $\gamma_{\varepsilon}$ into several arcs, on each of which the second derivative of the Poincaré map preserves its sign. Geometrically this means that in that rectangle the graph has the form of a 'staircase' with steps of various width and height (Lemma 3.6).

The maximal number of points where the diagonal $\mathcal{D}$ intersects the graph $\gamma_{\varepsilon}$ is bounded above by the number of neutral points (Rolle's theorem). When $\mathcal{D}$ passes through the next neutral point in turn, the next pair of canard cycles in turn is either born or destroyed: how many there can be altogether depends on the mutual position of the neutral points (on the width and height of the 'steps').

The information on the derivatives of the Poincaré map we need to analyse the neutral points is obtained from the following geometric considerations. We consider the base strip in the phase space bounded by the vertical circles passing through the right-most and left-most jump points and containing the slow curve $M$.

Any trajectory passing through a fixed point of this strip $w \notin M$, in forward time hits a stable part of the slow curve, goes to the right along this part to a jump point, undergoes a jump, and then it either exits the base strip or hits another stable part and moves along it to the next jump point; the process repeats until it exits the strip. In reverse time the trajectory hits an unstable part of the slow curve, moves to the left along it to a reverse jump point, undergoes a reverse jump, and either exits the base strip or hits another unstable part, and so on, until it exits the strip. Thus, the trajectory passes 
near some arcs of the stable and unstable parts of the slow curve (see Figure 1 and the construction in $\$ 5.2$.

At the point $w$ a switch happens called a 'canard jump': to the left of this point the trajectory moves near stable segments, and to the right near unstable. When it moves near stable (unstable) segments, the convergence (scattering) of trajectories accumulates. The derivative of the Poincaré map is determined by the sum of the integrals $\int f_{x}^{\prime}(x, y, 0) d y$ (cf. (2.4)) over those arcs (see Lemma 5.4, as well as the notation introduced in (5.3)).

Consider the trajectory passing through a point $(x,-\pi) \in \Gamma$. This trajectory undergoes at most one 'canard jump'. Depending on its direction (downwards or upwards), the canard jump shifts to the right or to the left as $x$ increases. In the first case the derivative of the Poincaré map increases, since time increases while the trajectory passes near unstable parts of the slow curve. Similarly, in the second case the derivative of the Poincaré map decreases. Switching between the increasing regime and the decreasing regime happens when the canard jump reaches some fold point on the slow curve (see the heuristic description in $\$ 5.1$.

Thus, the derivative of the Poincaré map is piecewise monotonic, and the number of intervals of monotonicity does not exceed the number of fold points of the slow curve (see Lemma 3.5 and its proof in \$5). This proves that the number of neutral points (and therefore also of canard cycles) does not exceed the number of fold points. An explicit construction (see 9 ) can be used to show that the maximal number of canard cycles is realized on an open set of systems (Theorem 2.2).

3.2. Structure of the proof. The proof of the main results is divided into a series of lemmas, which we state in the following subsection. Lemmas 3.1 (on the graph) and 3.2 (on monotonicity) repeat the analogous lemmas in [3, 5, almost word for word (there are insignificant modifications). They enable us to prove parts 1-4 of Theorem 2.5 for $k=1$ (that is, to prove the existence of attracting canard cycles for small values of the parameters). This part of the proof hardly differs from the proof of the analogous assertions for the case of a convex slow curve. Lemmas 3.5 and 3.6 enable us to prove the main results (Theorems 2.5] and 2.7) in full generality. $\$ 3.4$ is devoted to deriving these theorems from the lemmas stated before.

In 4 we present the requisite facts about normal forms and estimates for the derivatives of the Poincaré map.

The more subtle analysis that enables us to prove Lemmas 3.5 and 3.6 and, using them, to estimate the number of canard cycles being born and to prove part 5 of Theorem 2.5. as well as Theorem 2.7 requires us to obtain estimates for the second derivative of the Poincaré map. This analysis is substantially different from the convex case and constitutes the main contents of this paper (\$5). We consider the effects related to nonconvexity by using a simple example (see the heuristic description in 5.1). Then we analyse the general case and prove Theorems 2.5 and 2.7 (see $\$ \$ 5.255 .4$ ).

In $\sqrt{6}$ we prove Theorem 2.2

The technical assertions we need to estimate the derivatives of the Poincaré map are proved in $₫ 7$.

3.3. Basic facts and notation. In this subsection we introduce some notation we will use in the paper and state several lemmas describing the properties of the Poincaré map.

The following lemma formalizes the assertion that the graph of the Poincaré map is contained in the union of two narrow strips (see Figure 5).

Lemma 3.1 (the graph). There exist constants $c_{1,2}^{ \pm}>0$ such that for any sufficiently small $\varepsilon>0$ there are intervals $D_{\varepsilon}^{+}$and $D_{\varepsilon}^{-}$in the inverse image and the image of the 
map $P_{\varepsilon}$, respectively, that have the following properties.

1. $\left|D_{\varepsilon}^{ \pm}\right|=O\left(e^{-c_{1}^{ \pm} / \varepsilon}\right)$.

2. $\mid P_{\varepsilon}^{\prime} \|_{S^{1} \backslash D_{\varepsilon}^{+}}=O\left(e^{-c_{2}^{+} / \varepsilon}\right)$.

3. $\mid\left(P_{\varepsilon}^{-1}\right)^{\prime} \|_{S^{1} \backslash D_{\varepsilon}^{-}}=O\left(e^{-c_{2}^{-} / \varepsilon}\right)$.

4. Consider the strips $\Pi_{\varepsilon}^{+}:=D_{\varepsilon}^{+} \times S^{1}$ and $\Pi_{\varepsilon}^{-}:=S^{1} \times D_{\varepsilon}^{-}$. The graph $\gamma_{\varepsilon}$ is contained in their union: $\gamma_{\varepsilon} \subset \Pi_{\varepsilon}^{+} \cup \Pi_{\varepsilon}^{-}$.

The proof of this lemma for the case of a convex slow curve was given in [5] and carries over to the general case without changes, since it does not use the fact of convexity in any essential way.

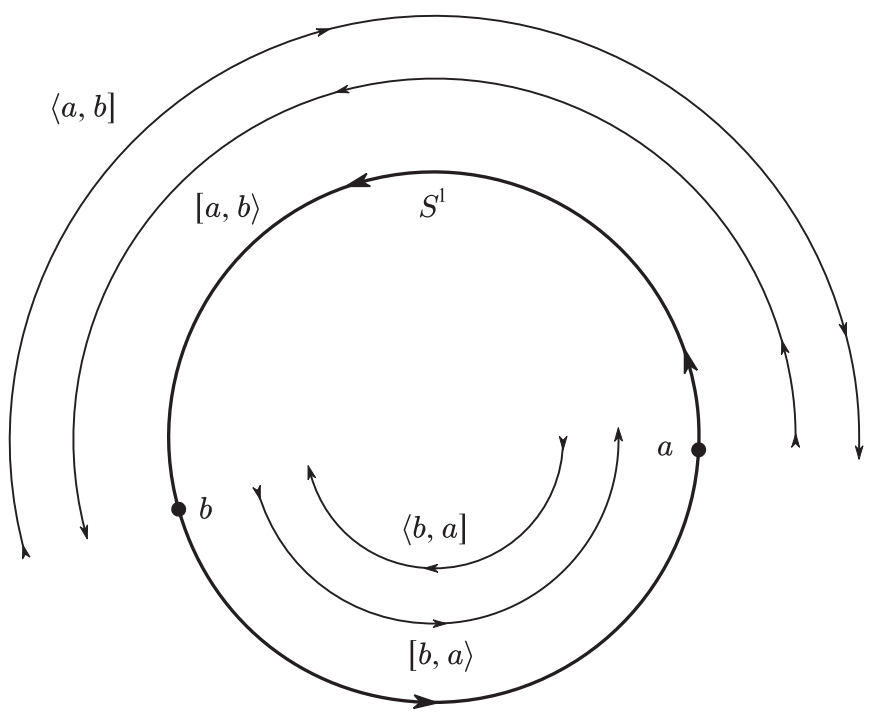

FiguRE 3. Orientation of arcs.

The following lemma formalizes the assertion that the graph $\gamma_{\varepsilon}$ moves monotonically to the left and upwards as $\varepsilon \rightarrow 0^{+}$. To state it we need to recall some notation used in 5.

Consider arbitrary points $a$ and $b$ on the oriented circle $S^{1}$. They divide the circle into two arcs. We denote by $[a, b\rangle$ the arc from the point $a$ to the point $b$ (in the sense of the orientation of the circle $S^{1}$ ) oriented in the same way as $S^{1}$. We denote by $\langle a, b]$ the same arc oriented in the opposite way (see Figure 3).

We denote by $P_{\varepsilon}^{[a, b\rangle}$ the Poincaré map along the phase curves of the system (2.1) from the transversal $y=a$ to the transversal $y=b$ in forward time (that is, along the arc $[a, b\rangle)$. We set $P_{\varepsilon}^{\langle a, b]}:=\left(P_{\varepsilon}^{[a, b\rangle}\right)^{-1}$. This map is the Poincaré map in the reverse time from the transversal $y=b$ to the transversal $y=a$, which is emphasized by the notation for the arc (the direction of the angular bracket indicates the direction of time).

In what follows, in all expressions containing the signs \pm , either only the upper signs or only the lower signs should be read.

Recall that $G^{ \pm}$denote the left-most $(+)$and the right-most $(-)$jump points (we call them the principal reverse and forward jump point, respectively; they are uniquely determined due to the nondegeneracy conditions; see \$2.2). Suppose they have coordinates $\left(\sigma^{ \pm}, \tau^{ \pm}\right)$, respectively (the slow curve $M$ is contained in the strip $\left\{\tau^{+} \leq y \leq \tau^{-}\right\}$). For 


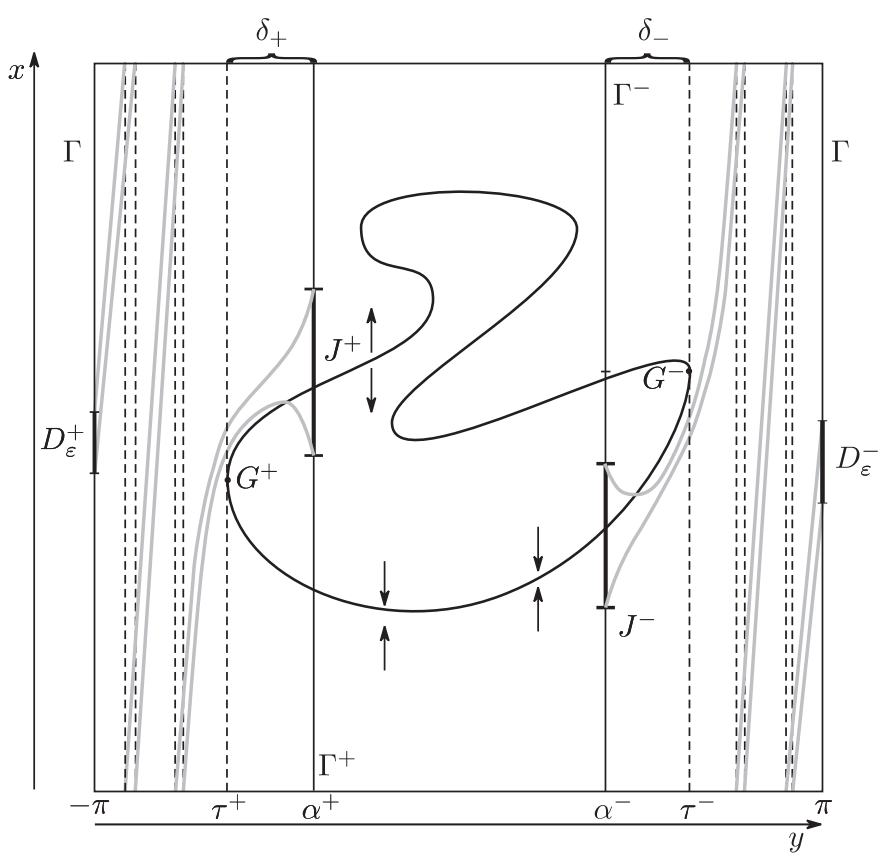

Figure 4. Nonconvex slow curve of a fast-slow system on the torus; the slow motion is directed horizontally.

some small fixed positive $\delta_{+}$and $\delta_{-}$we define the following objects:

1. The transversals $\Gamma^{ \pm}$intersecting the slow curve $M$ near the principal jump points (see Figure 4):

$$
\Gamma^{ \pm}:=S^{1} \times\left\{\alpha^{ \pm}\right\}
$$

where $\alpha^{ \pm}=\tau^{ \pm} \pm \delta_{ \pm}$.

2. Segments $J^{+}$and $J^{-}$of the transversals $\Gamma^{ \pm}$intersecting the unstable and stable parts of the slow curve, respectively.

3. The segments $D_{\varepsilon}^{ \pm}$, whose existence is asserted in Lemma 3.1

$$
D_{\varepsilon}^{+}:=P_{\varepsilon}^{\left\langle-\pi, \alpha^{+}\right]}\left(J^{+}\right), \quad D_{\varepsilon}^{-}:=P_{\varepsilon}^{\left[\alpha^{-}, \pi\right\rangle}\left(J^{-}\right), \quad D_{\varepsilon}^{ \pm}=\left[p_{\varepsilon}^{ \pm}, q_{\varepsilon}^{ \pm}\right\rangle .
$$

Thus, we have chosen the segment $J^{+}$which appears in Definition 2.4 of a canard solution; this completes the definition. All the trajectories intersecting the segment $D_{\varepsilon}^{+}$also intersect $J^{+}$and therefore are canard.

We further denote by $A_{\varepsilon}^{+}$and $A_{\varepsilon}^{-}$the points of the graph $\gamma_{\varepsilon}$ that lie over the endpoints of the segment $D_{\varepsilon}^{+}$(see Figure 5):

$$
A_{\varepsilon}^{-}:=\left(p_{\varepsilon}^{+}, P_{\varepsilon}\left(p_{\varepsilon}^{+}\right)\right), \quad A_{\varepsilon}^{+}:=\left(q_{\varepsilon}^{+}, P_{\varepsilon}\left(q_{\varepsilon}^{+}\right)\right),
$$

and by $E_{\varepsilon}^{-}$and $E_{\varepsilon}^{+}$, the upper left and the lower right corners of the rectangle $K_{\varepsilon}:=$ $D_{\varepsilon}^{+} \times D_{\varepsilon}^{-}$:

$$
E_{\varepsilon}^{-}:=\left(p_{\varepsilon}^{+}, q_{\varepsilon}^{-}\right), \quad E_{\varepsilon}^{+}:=\left(q_{\varepsilon}^{+}, p_{\varepsilon}^{-}\right) .
$$

Let $\bar{C}_{\varepsilon}$ be the lift to the universal cover of the torus of the point $C_{\varepsilon}$, which depends continuously on $\varepsilon$ (here $C_{\varepsilon}$ means one of the points $A_{\varepsilon}^{ \pm}, E_{\varepsilon}^{ \pm}$defined above). We also denote $\Delta(\bar{C}):=y(\bar{C})-x(\bar{C})$ for some point $\bar{C}$ on the universal cover of the torus. 


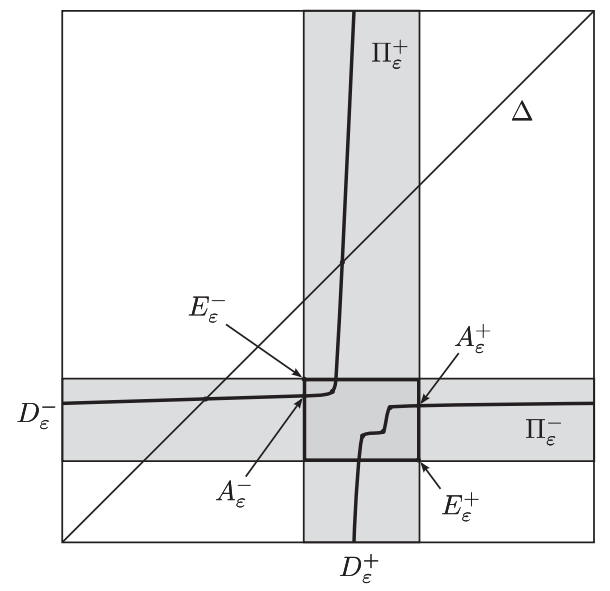

Figure 5. The graph of the Poincaré map.

Lemma 3.2 (monotonicity). The following hold:

1. $\frac{d}{d \varepsilon}(x-y)\left(\bar{C}_{\varepsilon}\right) \rightarrow+\infty$ as $\varepsilon \rightarrow 0^{+}$for every choice of $\bar{C}_{\varepsilon}=\bar{A}_{\varepsilon}^{ \pm} ; \bar{E}_{\varepsilon}^{ \pm}$.

2. The equation $\Delta\left(\bar{E}_{\varepsilon}^{-}\right)=2 \pi n$ has a root $\varepsilon=\varepsilon_{n}$ for every $n$, and $\varepsilon_{n}=O(1 / n)$.

Remark 3.3. It follows from the second part of the lemma that for $\varepsilon=\varepsilon_{n}$ the diagonal

$$
\mathcal{D}:=\{y=x \quad(\bmod 2 \pi \mathbb{Z})\}
$$

passes through the upper left corner of the rectangle $K_{\varepsilon}$.

The proof of Lemma 3.2 for the case of a convex slow curve given in [5] carries over to the general case without any changes.

Definition 3.4. A point $x$ is called neutral for the Poincaré map $P_{\varepsilon}$ if $P_{\varepsilon}^{\prime}(x)=1$. The corresponding point of the graph $\gamma_{\varepsilon}$ of the Poincaré map is also called neutral.

The geometric meaning of the following lemma is as follows: the graph of the Poincaré map near neutral points has the form of a 'staircase' (see Figure 6). Below we show (see Lemma 5.5) that the relative logarithmic sizes of its 'steps' do not change as $\varepsilon$ decreases.

Lemma 3.5 (on the staircase). Under the hypotheses of Theorem 2.5, for some positive integer $K$ the following hold.

1. The set $U:=\left\{x \in \Gamma \mid P_{\varepsilon}^{\prime}(x) \in[1 / 2,2]\right\}$ is a union of $2 K$ disjoint arcs; the second derivative $P_{\varepsilon}^{\prime \prime}$ preserves its sign on each of them and changes sign when passing from one arc to a neighbouring one.

2. The number $2 K$ does not exceed the total number $2 N$ of fold points of the slow curve.

3. There is exactly one neutral point on each of these arcs. We number them cyclically and denote them consecutively as $\xi_{i}, i=1, \ldots, 2 K$. To simplify the notation we also set $\xi_{i+2 K} \equiv \xi_{i}$.

4. We have $U \subset D_{\varepsilon}^{+}$and all the points $\xi_{i}$ lie inside the rectangle $K_{\varepsilon}$.

5. For any two points $\xi_{i}, \xi_{j}, \xi_{i} \neq \xi_{j}$, the expression $\Delta\left(\xi_{i}\right)-\Delta\left(\xi_{j}\right)$ is nonzero and its sign is independent of $\varepsilon$.

6. For any point $\xi_{i}$ we have $\pm\left(\Delta\left(\bar{A}_{\varepsilon}^{ \pm}\right)-\Delta\left(\xi_{i}\right)\right)>0$.

Lemma 3.6 (the step). Under the hypotheses of Theorem 2.7 the number $2 K$ in the preceding lemma takes the maximal value 4 . 


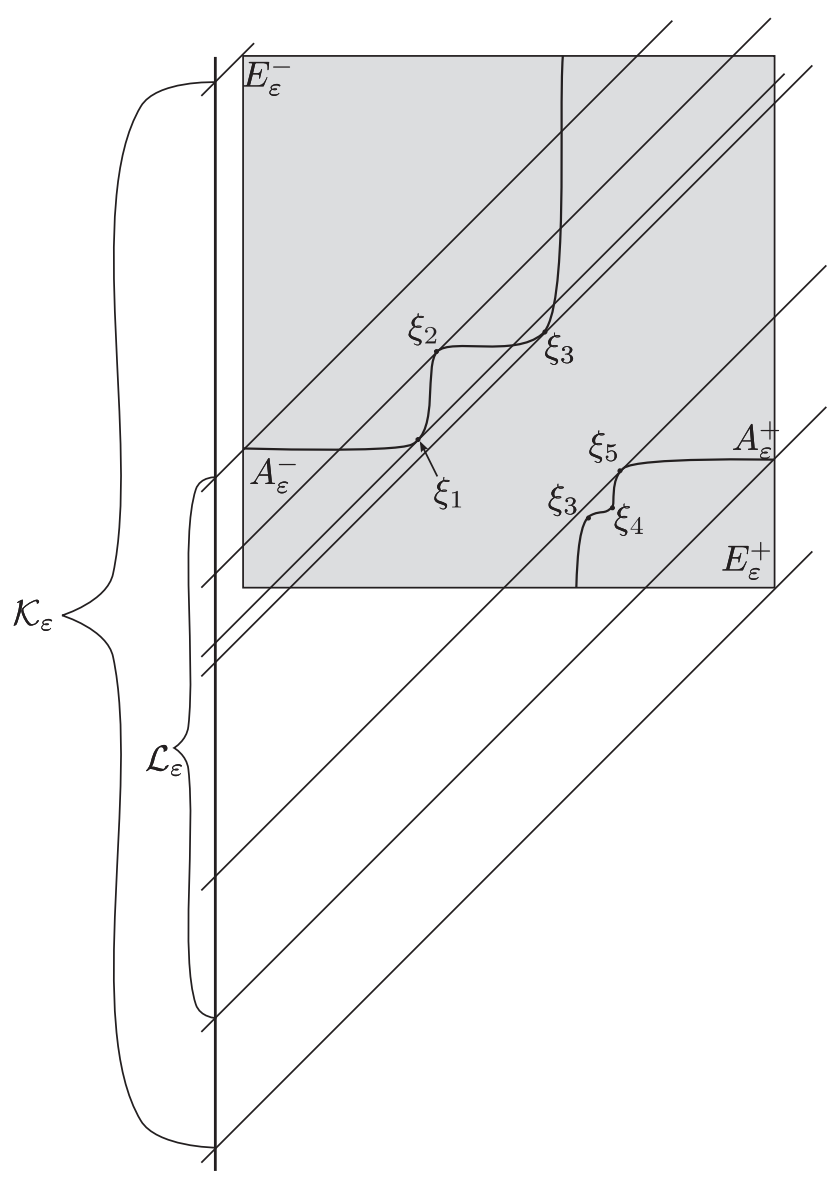

FiguRE 6 . The rectangle $K_{\varepsilon}$ (shaded) and the neutral points on the graph of the Poincaré map.

Lemmas 3.5 and 3.6 will be proved in $\$ 5$.

3.4. Domains on the straight line $\varepsilon$. In this subsection we derive Theorems 2.7 and 2.5 from the lemmas stated in the preceding subsection.

Proof of Theorem 2.5. We define segments $R_{n}$ as follows:

$$
R_{n}:=\left\{\varepsilon \in\left(\mathbb{R}_{+}, 0\right) \mid \Delta\left(\bar{E}_{\varepsilon}^{+}\right) \leq 2 \pi n \leq \Delta\left(\bar{E}_{\varepsilon}^{-}\right)\right\}=\left[\alpha_{n}, \beta_{n}\right] .
$$

With $R_{n}$ thus defined, it follows from Lemmas 3.1 and 3.2 that parts 1, 2, and 3 of Theorem 2.5 hold. The proof of this fact carries over from [5] without changes.

Consider the function $\varphi_{\varepsilon}(x)=P_{\varepsilon}(x)-x$ and some lift of it $\bar{\varphi}_{\varepsilon}(x)$, which depends continuously on $\varepsilon$, to the universal cover. Consider the segments $\mathcal{K}_{\varepsilon}:=\Delta\left(\bar{K}_{\varepsilon}\right)=$ $\left[\Delta\left(\bar{E}_{\varepsilon}^{-}\right), \Delta\left(\bar{E}_{\varepsilon}^{+}\right)\right]$and $\mathcal{L}_{\varepsilon}:=\left[\Delta\left(\bar{A}_{\varepsilon}^{-}\right), \Delta\left(\bar{A}_{\varepsilon}^{+}\right)\right]$(see Figure [6). Obviously, $\mathcal{L}_{\varepsilon} \subset \mathcal{K}_{\varepsilon}$. By part 3 of Lemma 3.5, the derivative $\bar{\varphi}_{\varepsilon}^{\prime}(x)$ vanishes at the neutral points $x_{k}(\varepsilon)=x\left(\xi_{k}(\varepsilon)\right)$, $k=1, \ldots, 2 K$. By part 6 of the same lemma, $u_{k}(\varepsilon)=\bar{\varphi}_{\varepsilon}\left(x_{k}(\varepsilon)\right)=\Delta\left(\xi_{k}\right) \in \mathcal{L}_{\varepsilon}$. Part 5 of the same lemma implies that the order of the points $u_{k}(\varepsilon)$ does not change as $\varepsilon \rightarrow 0^{+}$. Thus the points $u_{k}(\varepsilon)$ divide the circle into $2 K$ intervals; we denote these intervals by $\mathcal{I}_{j}(\varepsilon), j=1, \ldots, 2 K$. By part 1 of Lemma 3.5, for every $b \in \mathcal{I}_{j}(\varepsilon)$ the function $\bar{\varphi}_{\varepsilon}(x)-b$ 
has a fixed even number of simple zeros for a given $j$; we denote it by $2 l_{j}$. When $b$ passes one of the points $u_{k}(\varepsilon)$, the number of zeros increases or decreases by two.

Proposition 3.7. There exists a unique $j$ such that $l_{j}=0$.

This proposition will be proved below. Applying it yields that $l_{j} \leq K$ for any $j$. We denote by $k$ the maximum of all the $l_{j}$. Then the number of roots of the equation $\bar{\varphi}_{\varepsilon}(x)=0$ (these correspond to fixed points of the map $P_{\varepsilon}$ ) does not exceed $2 k$, which proves part 5 of Theorem 2.5. For every $i \leq k$ there exists an interval $\mathcal{I}_{j}$ such that $l_{j}=i$.

It follows from part 2 of Lemma 3.2 on monotonicity that for $\varepsilon=\varepsilon_{n}=O(1 / n)$ the upper endpoint of the segment $\mathcal{K}_{\varepsilon}$ passes through the point $2 \pi n$. We set

$$
\widetilde{C}_{n}^{i}:=\left\{\varepsilon \in R_{n} \mid \mathcal{I}_{j}(\varepsilon) \cap \mathcal{L}_{\varepsilon} \ni 2 \pi n, l_{j}=i\right\}, \quad i=1, \ldots, k .
$$

Taking account of the continuity properties, the sets $\widetilde{C}_{n}^{i}$ are nonempty and open. We choose an interval $C_{n}^{i}$ in each of them. By construction, for every $\varepsilon \in C_{n}^{i}$ the equation $\bar{\varphi}_{\varepsilon}(x)=0$ has $2 i$ roots, which are fixed points for the map $P_{\varepsilon}$. Since $2 \pi n \in \mathcal{L}_{\varepsilon}$, the diagonal $\mathcal{D}$ intersects the graph $\gamma_{\varepsilon}$ in points lying over the segment $D_{\varepsilon}^{+}$. Consequently, all the fixed points of the Poincaré map correspond to closed canard solutions, and we have proved part 4 of Theorem 2.5 for the $C_{n}^{i}$ defined here and for this $k$. This completes the proof of the theorem.

Taken together with Proposition 2.6 (which we will prove in $\$ 5.5$ ), this gives a proof of Theorem 2.1

Proof of Theorem 2.7. It follows from Lemma 3.6, as well as from Proposition 3.7, that under the hypotheses of Theorem 2.7 the circle is divided into 4 intervals $\mathcal{I}_{j}$, and consequently, the number $k$ in the statement of Theorem 2.5 is equal to 2. Indeed, under the passage to a neighbouring interval the number $l_{j}$ changes by 1 , and therefore if $l_{j}=0$, then $l_{j+2}=0$ or $l_{j+2}=2$. But the first case is impossible by Proposition 3.7. This proves Theorem 2.7 .

Proof of Proposition 3.7. By choosing coordinates (different ones in the image and preimage), without loss of generality we set $P_{\varepsilon}(0)=0$ and consider the fundamental square $[0,2 \pi]^{2}$. Then the graph $\gamma_{\varepsilon}$ becomes the graph of a monotonically increasing function which connects the lower left-hand corner of the fundamental square with the upper right-hand one. Consider points $A, B \in \gamma_{\varepsilon}$. We assume without loss of generality that $x(B)>x(A)$, and consequently (by monotonicity), $y(B)>y(A)$. We claim that $\mid \Delta(A)-$ $\Delta(B) \mid<2 \pi$. Indeed:

$$
\begin{aligned}
|\Delta(A)-\Delta(B)| & =|(y-x)(A)-(y-x)(B)| \\
& =|(x(B)-x(A))-(y(B)-y(A))| \\
& \leq \max (x(B)-x(A), y(B)-y(A)) \\
& \leq \max (x(B), y(B)) \\
& <2 \pi .
\end{aligned}
$$

It follows from (3.3) and continuity arguments that $\Delta\left(\gamma_{\varepsilon}\right)$ is a segment whose length is less than $2 \pi$. By projecting $\Delta\left(\gamma_{\varepsilon}\right)$ onto the circle $\mathbb{R} / 2 \pi \mathbb{Z}$, we obtain an arc. Denoting the complement of this arc by $\mathcal{I}$, we have: for every $b$ in $\mathcal{I}$ (and only for them!) the diagonal $\Delta(x, y)=b$ does not intersect $\gamma_{\varepsilon}$, that is, $\varphi_{\varepsilon}(x)=b$ has no solutions. Since the maximum and minimum of $\left.\Delta\right|_{\gamma_{\varepsilon}}$ are attained at the points $\xi_{k}$, the interval $\mathcal{I}$ is represented in the form $\mathcal{I}=\left[\Delta\left(\xi_{i}\right), \Delta\left(\xi_{j}\right)\right]$ for some $i, j$. There are no other points $\Delta\left(\xi_{k}\right)$ on the interval. Consequently, $\mathcal{I}$ coincides with one of the intervals $\mathcal{I}_{k}$ for some $k$. 


\section{Normalization AND EStimates FOR DERIVATiVES}

In this section we state the theorems on fast-slow systems that we need to estimate the derivative of various Poincaré maps. Essentially, this section just reproduces the section "Normalization" in [5].

For what follows we shall need two theorems on normal forms of fast-slow systems on the torus that were proved by Guckenheimer and Ilyashenko.

4.1. Nonlinear rotation. The following theorem describes the structure of fast-slow dynamics on the torus outside a neighbourhood of the slow curve.

Theorem 4.1 ([3]). Consider the vector field on the cylinder $x \in S^{1}=\mathbb{R} / \mathbb{Z}, y \in \mathbb{R}$ defined by the system

$$
\dot{x}=f(x, y, \varepsilon), \quad \dot{y}=\varepsilon g(x, y, \varepsilon), \quad f>0, g>0 .
$$

Let $a, b \in \mathbb{R}, a<b$, and $\varepsilon>0$. Then the Poincaré map $P_{\varepsilon}^{[a, b\rangle}$ from $\Gamma^{a}=\{y=a\}$ to $\Gamma^{b}=\{y=b\}$ has the form

$$
P_{\varepsilon}^{[a, b\rangle}(x)=G_{\varepsilon}^{1} \circ\left(G_{\varepsilon}^{2}(x)+T(\varepsilon)\right),
$$

where $T(\varepsilon) \rightarrow \infty$ and $G_{\varepsilon}^{1,2}$ are diffeomorphisms of the circle, $G_{\varepsilon}^{1,2} \rightarrow G_{1,2}$ as $\varepsilon \rightarrow 0^{+}$, and $G_{1,2}$ are also diffeomorphisms of the circle.

In other words, the correspondence map from a fixed vertical transversal to a fixed vertical transversal can be reduced by a change of coordinates in the image and preimage to a rotation through an angle $T(\varepsilon)$ that tends to infinity as $\varepsilon \rightarrow 0^{+}$. It is important to point out that the corresponding changes of coordinates have bounded derivatives as $\varepsilon \rightarrow 0^{+}$and tend to smooth changes of coordinates.

Theorem 4.1 was proved in [3, Theorem 2, p. 35].

4.2. Normalization near the slow curve. The following theorem shows that near the slow curve outside a neighbourhood of a jump point the system (2.1) is smoothly equivalent to a linear system.

Theorem 4.2 ([3]). Consider the system

$$
\dot{x}=f(x, y, \varepsilon), \quad \dot{y}=\varepsilon g(x, y, \varepsilon), \quad g>0 .
$$

Suppose that the corresponding fast system has a curve of hyperbolic fixed points (a nondegenerate slow curve). Then for small $\varepsilon>0$ in a neighbourhood of the curve of fixed points (outside fixed neighbourhoods of jump points) the system is smoothly orbitally equivalent to the family

$$
\dot{x}=a(y, \varepsilon) x, \quad \dot{y}=\varepsilon .
$$

The proof of this theorem can be found in [3, Theorem 3, p.38]. It follows from Fenichel's theorem (see [6], and also [7] and [8]) that in a neighbourhood of the stable (unstable) part of the slow curve $M^{-}\left(M^{+}\right)$there exists a smooth invariant manifold $S_{\varepsilon}^{-}$ $\left(S_{\varepsilon}^{+}\right)$that can be represented as the graph of a function $x=s^{-}(y, \varepsilon)\left(x=s^{+}(y, \varepsilon)\right)$. In the normalizing coordinates, this manifold, which is called the true slow curve, is given by the equation $x=0$. It follows from the variational equation that the function $a(y, \varepsilon)$ in Theorem 4.2 has the form

$$
a(y, \varepsilon)=f_{x}^{\prime}(s(y, \varepsilon), y, \varepsilon) .
$$

Remark 4.3. Actually, the true slow curves $S_{\varepsilon}^{ \pm}$are not uniquely determined. However, the distance between such curves is of order $O\left(e^{-C / \varepsilon}\right)$ (that is, they are exponentially close), and we can choose any of them. 
4.3. The Poincaré map near a jump point. We state one more result, which will let us estimate the derivative of the Poincaré map near a jump point very roughly.

Remark 4.4. In what follows the letter $C$ (without indices) will denote positive constants independent of $\varepsilon$. They can be different in different formulae.

Lemma 4.5. Consider the fast-slow system

$$
\dot{x}=f(x, y, \varepsilon), \quad \dot{y}=\varepsilon, \quad(x, y) \in S^{1} \times \mathbb{R} .
$$

Then for sufficiently small $\varepsilon$ the Poincaré map $P_{\varepsilon}^{[a, b\rangle}: \Gamma^{a} \rightarrow \Gamma^{b}$ satisfies the estimate

$$
\left|\ln \left(P_{\varepsilon}^{[a, b\rangle}(x)\right)^{\prime}\right|<C \frac{b-a}{\varepsilon} .
$$

Proof. Let $x=x\left(y ; x_{0}, \varepsilon\right)$ be the phase curve passing through a point $\left(x_{0}, a\right)$ for a given $\varepsilon$. Since $f_{x}^{\prime}$ is bounded on the torus (above and below), it follows from the variational equation with respect to the initial conditions that

$$
\left(P_{\varepsilon}^{[a, b\rangle}\left(x_{0}\right)\right)^{\prime}=\exp \left(\frac{1}{\varepsilon} \int_{a}^{b} f_{x}^{\prime}\left(x\left(y ; x_{0}, \varepsilon\right), y, \varepsilon\right) d y\right)<e^{\frac{C}{\varepsilon}(b-a)} .
$$

A similar estimate holds for the inverse map. By taking the logarithm we obtain the required estimate for the absolute value.

This lemma will be used to obtain rough estimates for the derivatives in a neighbourhood of a jump point. A more precise analysis of the behaviour of the system in a neighbourhood of a jump point is given by the following theorem.

Theorem 4.6 ([5, Theorem 4.3]). In the notation of \$3.3 (see $p$.182), let $T^{ \pm}=J^{ \pm} \cap M$. Then for some $\lambda>0$ the following holds:

$$
\begin{aligned}
\ln \left(\frac{d}{d x} P_{\varepsilon}^{\left\langle-\pi, \alpha^{+}\right]}(x)\right) & =\frac{\Phi\left(T^{+} G^{+}\right)+O\left(\varepsilon^{\lambda}\right)}{\varepsilon}, \quad x \in J^{+}, \\
\ln \left(\frac{d}{d x} P_{\varepsilon}^{\left[\alpha^{-}, \pi\right\rangle}(x)\right) & =\frac{\Phi\left(T^{-} G^{-}\right)+O\left(\varepsilon^{\lambda}\right)}{\varepsilon}, \quad x \in J^{-},
\end{aligned}
$$

where $O\left(\varepsilon^{\lambda}\right)$ is uniform with respect to $x$.

\section{An estimate for the SeCond Derivative of the Poincaré MaP}

In this section we prove Lemmas 3.5 and 3.6 .

5.1. Heuristic description. We consider a system of the form (2.1) corresponding to Figure 1 (see p. 177). In this subsection we describe its dynamics heuristically and show the reasons for two pairs of canard solutions to appear under the balancing conditions.

Recall (see 44.2 ) that in a neighbourhood of every part of the slow curve $M$ consisting of regular singular points of the fast motion, for small $\varepsilon$ there exists an invariant manifold that is $O(\varepsilon)$-close to the corresponding part of $M$, which is called the true slow curve. For a fixed part of the slow curve, the true slow curves are not uniquely determined, but they are exponentially close to one another, and we can choose any of them. Consider maximal true slow curves corresponding to the parts $G^{+} E, E D$, and $D G^{-}$. We denote them by $S_{1}$, $S_{2}$, and $S_{3}$, respectively. We extend them beyond the corresponding jump points (forward and backward in time; see Figure 7). We introduce the notation: $u^{j}:=S_{j} \cap J^{+}, j=1,2,3$. Note that the pairwise distances between the points $u^{j}$ are exponentially small.

We consider the trajectory passing through a point $u \in J^{+}$. Recall that the dynamics in a neighbourhood of a part of the slow curve is described by a linear equation of the form (4.4). In the case of an unstable part this means that the trajectory spends some 


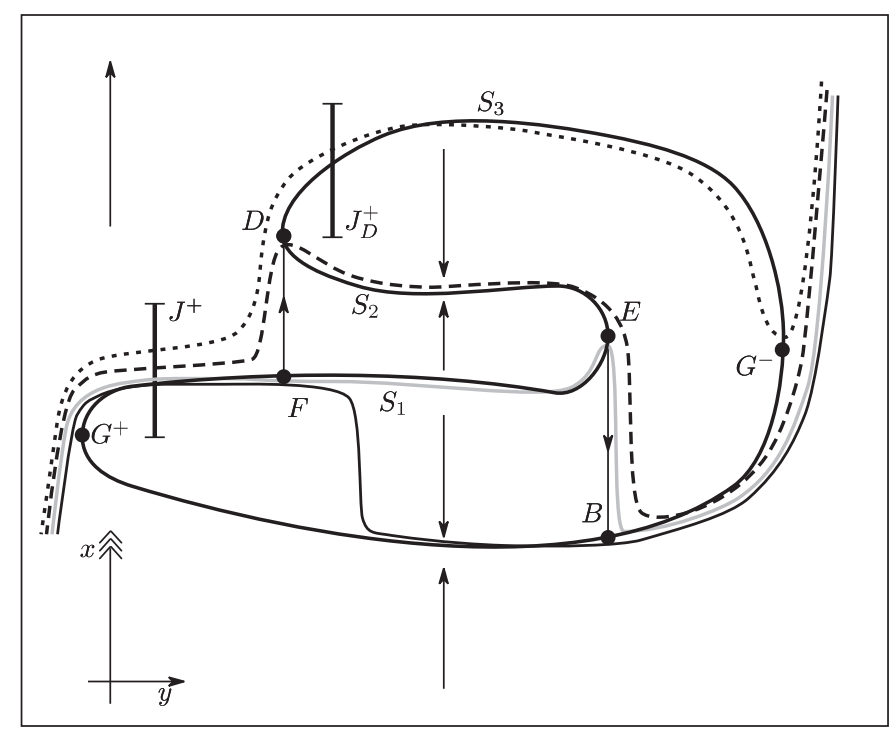

Figure 7. True slow curves in a system with two folds: the scale is considerably distorted for illustration.

time near the true slow curve (written as $x=0$ in the normalizing coordinates), after which it leaves the neighbourhood of the unstable part of the slow curve and in time $O(\varepsilon)$ again enters a neighbourhood of the slow curve. Here two variants are possible:

1. The trajectory enters a neighbourhood of a stable part of the slow curve and then moves along it; we call this phenomenon a canard jump.

2. The trajectory enters a neighbourhood of a reverse jump point and undergoes a reverse jump, then moves for some time near an unstable part of the slow curve (see Figure [7] the trajectories passing near $u^{3}$ and intersecting $J_{D}^{+}$are of this type).

It follows from the normal form (4.4) that, as the trajectory moves in a neighbourhood of an unstable part of the slow curve, the derivative of the solution with respect to the initial condition $u$ increases ('scattering' is accumulated), and as the trajectory moves along a stable part it decreases ('contraction' is accumulated). By the chain rule, the derivatives on different parts of the trajectory, mentioned above, contribute to the derivative of the Poincaré map $P_{\varepsilon}$. Thus, to estimate the second derivative of the Poincaré map it is necessary to examine what proportion of the time the trajectory spends near stable parts of the slow curve, and what proportion near unstable parts.

The following cases are possible.

1. $u<u^{1}$. As the initial condition approaches $u^{1}$ from below, the time spent by the trajectory near the stable part $G^{+} E$ increases by comparison with the time spent near the unstable part $G^{-} G^{+}$. Thus, the derivative increases and attains its maximum at the point $u^{1}$ (in this case the trajectory coincides with the true slow curve $\left.S_{1}\right)$.

2. $u \in\left(u^{1}, u^{2}\right)$. As the initial condition moves from $u^{1}$ to $u^{2}$, the trajectory spends less time in a neighbourhood of the unstable part $G^{+} E$ by comparison with the stable part $E D$. The derivative decreases and attains its minimum at the point $u^{2}$.

3. $u \in\left(u^{2}, u^{3}\right)$. As the initial condition moves from $u^{2}$ to $u^{3}$, the trajectory spends more time in a neighbourhood of the unstable part $D G^{-}$by comparison with 
the unstable part $E D$. The derivative increases and attains its maximum at the point $u^{3}$.

4. $u>u^{3}$. As the initial condition moves away from $u^{3}$, the trajectory spends less time in a neighbourhood of the unstable part $D G^{-}$by comparison with the stable part $G^{-} G^{+}$. The derivative decreases up to an exponentially small quantity.

It is easy to see that if the derivative $P_{\varepsilon}$ is greater than 1 for the trajectory passing through $u^{1}$, and it is less than 1 for the trajectory passing through $u^{2}$, then there exist four points at which this derivative takes the value 1 . Indeed, at the endpoints of the interval $J^{+}$this derivative is exponentially small, since the corresponding trajectories spend almost all the time near stable parts of the slow curve. On the other hand, near the point $u^{3}$ this derivative is exponentially large, since the trajectory spends almost all the time near unstable parts of the slow curve. Thus, on each of the intervals 1-4 described above there exists a neutral point. This condition is necessary and sufficient for Lemmas 3.5 and 3.6 to hold in this case.

As we shall show below (see Lemma 5.4 in the next subsection), the logarithmic derivative of the Poincaré map is determined by the sum of integrals of the form (2.4) over the corresponding arcs of the slow curve near which the trajectory passes. Thus, the conditions stated in the preceding paragraph can be reformulated in the form of the balancing conditions in Theorem 2.7

5.2. Limit behaviour of trajectories and estimates for derivatives. In this subsection we carry out certain necessary constructions and state several auxiliary assertions about estimating the derivatives of the Poincaré map. They let us formalize the heuristic description given in the preceding subsection.

Let $y^{*} \in\left[y\left(G^{+}\right), y\left(G^{-}\right)\right\rangle$. We consider the trajectory passing through a point $w:=$ $\left(x^{*}, y^{*}\right) \notin M$. Let it be given by the equation $x=x(y ; w)$. In reverse (forward) time, over time of order $O(\varepsilon)$ this trajectory is attracted to some unstable (stable) part of the slow curve $M$ after which, in view of the normal form (4.4), it moves slowly along this stable part to the left (to the right) to a neighbourhood of a jump point.

If the jump point reached after a reverse (forward) jump does not coincide with $G^{+}$ $\left(G^{-}\right)$, the trajectory is attracted to some other unstable (stable) part of the slow curve, and then the process continues until a neighbourhood of $G^{+}\left(G^{-}\right)$is reached. Then, after a reverse (forward) jump, the trajectory leaves the neighbourhood of the slow curve and, performing a large number of revolutions, reaches the transversal $\Gamma=\{y=-\pi\}=$ $\{y=\pi\}$ at the point with coordinate $x_{0}=x(-\pi ; w)\left(P_{\varepsilon}\left(x_{0}\right)\right)$. In terms of the preceding subsection, this trajectory undergoes a canard jump at the point $w$.

The dynamics we have just described, of the trajectory emitted from some point, motivates the following definition.

Definition 5.1. Suppose that a point $w=\left(x^{*}, y^{*}\right)$ does not lie on the slow curve (see Figure 8). We emit from $w$ the vertical rays in the direction of fast motion, and in the opposite direction, until intersection with the slow curve $M$. We denote the intersection points by $F^{-}:=F^{-}(w)$ and $F^{+}:=F^{+}(w)$, respectively; henceforth the sign $(-)$ corresponds to forward time, and the sign $(+)$ to the reverse. Consider the arc of the slow curve $M$ from the point $F^{-}\left(F^{+}\right)$to a forward (reverse) jump point. We denote the second endpoint of this arc by $G^{ \pm}:=G^{ \pm}(w)$. We denote this arc by $\left[F^{-}, G^{-}\right\rangle$ $\left(\left[G^{+}, F^{+}\right\rangle\right)$; the orientation is positive with respect to the direction of the $y$-axis. We define the contour $Z(w)$ corresponding to the point $w$ to be the following set of arcs of $M$ :

$$
Z(w):=\underbrace{\left[G_{l}^{+}, F_{l}^{+}\right\rangle+\cdots+\left[G_{1}^{+}, F_{1}^{+}\right\rangle}_{Z^{+}(w)}+\underbrace{\left[F_{1}^{-}, G_{1}^{-}\right\rangle+\cdots+\left[F_{m}^{-}, G_{m}^{-}\right\rangle}_{Z^{-}(w)},
$$


where

$$
\begin{aligned}
F_{i+1}^{ \pm} & =F^{ \pm}\left(G_{i}^{ \pm}\right), & G_{i+1}^{ \pm} & =G^{ \pm}\left(G_{i}^{ \pm}\right), \\
F_{1}^{ \pm} & =F^{ \pm}(w), & G_{1}^{ \pm} & =G^{ \pm}(w), \\
G_{l}^{+} & =G^{+}, & G_{m}^{-} & =G^{-} .
\end{aligned}
$$

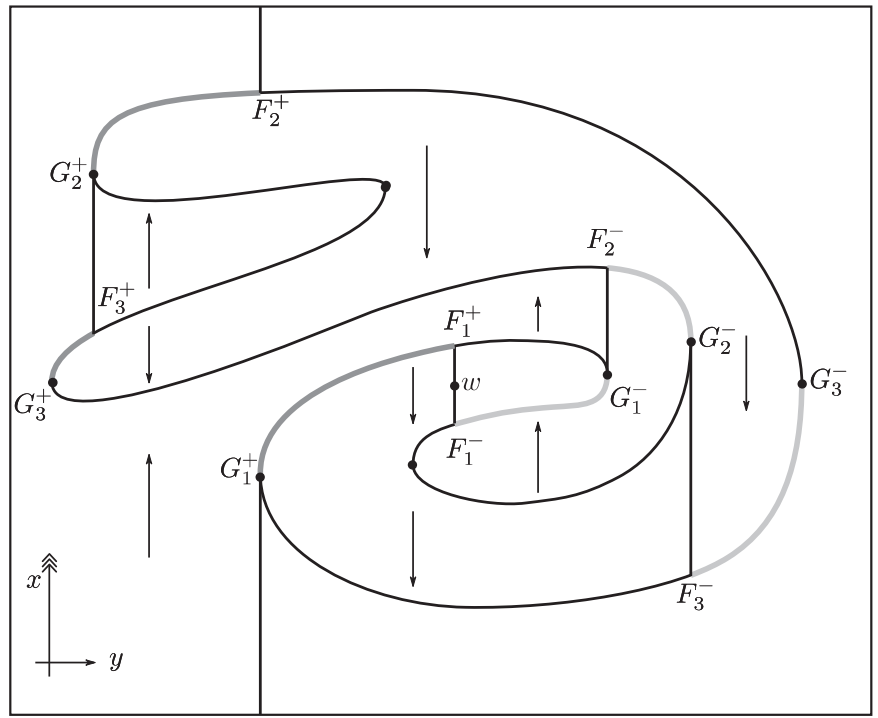

Figure 8 . The contour of a point $w$ for a system with a complicated nonconvex slow curve.

Using traditional terminology (see [9]) we can say that a contour is the union of the nonvertical parts of the corresponding singular trajectory. The contour $Z^{-}(w)\left(Z^{+}(w)\right)$ consists of the arcs of $M$ near which the trajectory emitted from the point $w$ spends almost all the time, until a forward (reverse) jump at the points $G^{-}\left(G^{+}\right)$. We set

$$
\begin{gathered}
\Phi^{ \pm}(w):=\Phi\left(Z^{ \pm}(w)\right)=\int_{Z^{ \pm}(w)} f_{x}^{\prime}(x, y) d y, \\
\Phi(w):=\Phi(Z(w))=\int_{Z(w)} f_{x}^{\prime}(x, y) d y .
\end{gathered}
$$

Definition 5.2. The value of the function $\Phi(Z(w))$ is called the integral of the contour $Z(w)$. A contour $Z(w)$ with zero integral $(\Phi(Z(w))=0)$ is called a neutral contour.

Below we shall show (see Lemma [5.6) that the derivative of the Poincaré map can be close to 1 if and only if the corresponding trajectory passes near a neutral contour. To formalize this assertion, we need to introduce an additional definition, which is motivated by the following observation: the value $\Phi(w)$ does not change if the point $w$ moves along a vertical segment without crossing the slow curve $M$.

We consider the set $\widehat{G}$ of jump points (forward and reverse). By the nondegeneracy conditions (2.2), this is a finite discrete set.

We impose the additional nondegeneracy condition

$$
\{y(z) \mid z \in \mathcal{G}: \Phi(z)=0\} \cap\{y(g) \mid g \in \widehat{G}\}=\varnothing
$$

on the system. In other words, the canard jumps of neutral contours do not lie over jump points. 
Recall that $U=\left\{x \in \Gamma \mid P^{\prime}(x) \in[1 / 2,2]\right\}$. Also recall that the vertical strip between the two principal jump points $\Pi:=S^{1} \times\left[y\left(G^{+}\right), y\left(G^{-}\right)\right]$is called the base strip. For every jump point apart from the principal ones, we also consider the maximal vertical interval containing this point and not intersecting $M$ in other points. We consider the union of $M$ with these intervals, and the complement of this union with respect to the base strip (see Figure 9):

$$
\begin{aligned}
& \Sigma:=M \cup \bigcup_{g \in \widehat{G}}\left(F^{+}(g), F^{-}(g)\right) \times\{y(g)\}, \\
& \bar{\Sigma}:=\Pi \backslash \Sigma,
\end{aligned}
$$

where by the interval $\left(F^{+}(g), F^{-}(g)\right)$ we mean the one of the two arcs which does not contain points of $M$ other than $g$.

We point out that by the nondegeneracy conditions (2.2) the set $\bar{\Sigma}$ has finitely many connected components. We also fix some small $\delta$ and consider the $\delta$-neighbourhood $\Sigma_{\delta}$ of the set $\Sigma$. We set

$$
\bar{\Sigma}_{\delta}:=\Pi \backslash \Sigma_{\delta} .
$$

We require $\delta$ to be chosen sufficiently small so that the intersection of every connected component of $\Sigma$ with $\bar{W}$ is nonempty and intersects any vertical circle $y=$ const in a segment (or the empty set).

In $\bar{\Sigma}$ we identify points $(x, y)$ and $\left(x^{\prime}, y\right)$ if and only if $\left[x, x^{\prime}\right\rangle \times\{y\} \cap M=\varnothing$. The resulting set is denoted by $\mathcal{V}$. We introduce the topology induced from $\mathbb{T}$ in it and denote the set of connected components by $V$. This is a finite set (by the nondegeneracy conditions).

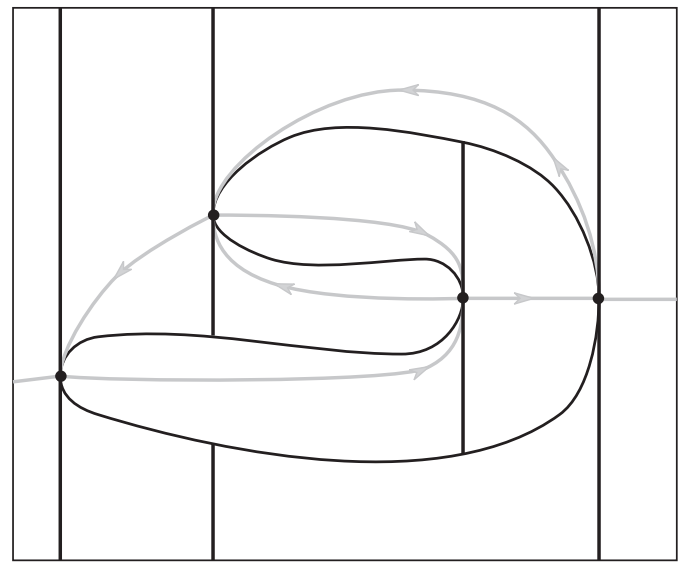

FiguRE 9. The graph $\mathcal{G}$ in the simplest nonconvex case.

It is easy to see that the pair $\mathcal{G}=(\widehat{G}, V)$ is a planar graph if we assume that points $g_{1}, g_{2} \in \widehat{G}$ are connected by an edge $v \in V$ if and only if there exists a representative of $v$ that is a smooth curve connecting these points. Thus, this graph admits natural embeddings into $\mathbb{T}$. We fix such an embedding (see Figure 9) by demanding that any nonempty intersection of any connected component of $\bar{\Sigma}_{\delta / 2}$ with a vertical segment contains a point in the corresponding arc of the graph. It is easy to see that the graph $\mathcal{G}$ thus embedded into $\mathbb{T}$ is a natural domain of definition of the function $\Phi(w)$. 
Lemma 5.3. For every point $w \in \bar{\Sigma}_{\delta}$ the following holds: the trajectory emitted from this point in forward (reverse) time enters $\Sigma_{\delta / 2}$ in time that is bounded above and does not leave this neighbourhood until it exits the strip $\Pi$.

We consider the trajectory passing through a point $x \in \Gamma$. Suppose that the trajectory intersects $\bar{\Sigma}_{\delta}$. In this case it also intersects some edge $v \in V$ at some point $z \in \bar{\Sigma}_{\delta / 2}$. By Lemma 5.3, as well as by the properties of the embedding of the edges $V$, this point is unique for sufficiently small $\varepsilon$. We denote it by $z(x)$. The function $z(x)$ may not be defined if the trajectory does not leave $\Sigma_{\delta}$ other than through the boundaries of the strip $\Pi$.

Lemma 5.4. For some $\lambda>0$ the following representations for the derivatives of the Poincaré maps hold at the points corresponding to the trajectory passing through $w=$ $\left(x^{*}, y^{*}\right) \in \bar{\Sigma}_{\delta}:$

$$
\begin{aligned}
\left.\log \frac{d}{d x} P_{\varepsilon}^{\left[y^{*}, \pi\right\rangle}\right|_{x=x^{*}} & =\frac{\Phi^{-}(w)+O\left(\varepsilon^{\lambda}\right)}{\varepsilon}, \\
\left.\log \frac{d}{d x} P_{\varepsilon}^{\left\langle\pi, y^{*}\right]}\right|_{x=x^{*}} & =-\frac{\Phi^{+}(w)+O\left(\varepsilon^{\lambda}\right)}{\varepsilon}, \\
\log \frac{d}{d x} P_{\varepsilon}\left(x_{0}\right) & =\frac{\Phi(w)+O\left(\varepsilon^{\lambda}\right)}{\varepsilon},
\end{aligned}
$$

where $O\left(\varepsilon^{\lambda}\right)$ is estimated uniformly with respect to $w$.

Lemma 5.5. Consider points $w_{1}, w_{2} \in \bar{\Sigma}_{\delta}$; suppose that the trajectories passing through them in reverse time intersect the transversal $\Gamma$ at points $x_{1}, x_{2}$, respectively. Let

$$
I^{ \pm}=I^{ \pm}\left(w_{1}, w_{2}\right):=\Phi\left(Z^{ \pm}\left(w_{1}\right) \cap Z^{ \pm}\left(w_{2}\right)\right) .
$$

Then

$$
\begin{aligned}
\left|x_{1}-x_{2}\right| & =O^{*}\left(\exp \left(\frac{-I^{+}+O\left(\varepsilon^{\lambda}\right)}{\varepsilon}\right)\right), \\
\left|P_{\varepsilon}\left(x_{1}\right)-P_{\varepsilon}\left(x_{2}\right)\right| & =O^{*}\left(\exp \left(\frac{I^{-}+O\left(\varepsilon^{\lambda}\right)}{\varepsilon}\right)\right) ;
\end{aligned}
$$

that is, the corresponding distances are asymptotically bounded above and below by the exponentially small expressions indicated above. The corresponding estimates are uniform with respect to $w_{1}, w_{2}$.

Lemma 5.6. For every $x \in U$ the point $z(x)$ is defined and there exists a point $z_{n}:=$ $z_{n}(x)$ on some edge of the graph $\mathcal{G}$ such that $\Phi\left(z_{n}\right)=0$ and

$$
\left|z(x)-z_{n}(x)\right|=O\left(\varepsilon^{\lambda}\right) .
$$

Lemma 5.7. For every $x \in U$,

$$
\operatorname{sgn}\left(P_{\varepsilon}^{\prime \prime}(x)\right)=-\operatorname{sgn}(f(z(x), 0)) .
$$

In other words, the sign of the second derivative of the Poincare map at neutral points is determined by the direction of the canard jump.

All the lemmas stated above will be proved below (see \$77).

5.3. Neutral points. In this subsection we derive parts 1 and 3-6 of Lemma 3.5 from the lemmas stated in the preceding subsection.

It is easy to see that the contour $Z(z)$ varies continuously as the point $z$ moves on some edge $v$ of the graph $\mathcal{G}$. This implies that the function $\Phi(z)$ is continuous on the edges of the graph. Moreover, $\Phi(z)$ is monotonic with respect to $y(z)$. Thus, on every edge $v$ there exists at most one zero of $\Phi(z)$ or, in other words, at most one 
neutral contour intersecting $v$. Together with Lemma 5.7, which proves that every neutral contour corresponds to exactly one neutral point, this gives parts 1 and 3 of Lemma 3.5. Part 4 follows from parts 2 and 3 of the lemma that deals with the graph, since outside $K_{\varepsilon}$ the derivative of the Poincaré map is exponentially small or large.

To prove part 5, we consider neutral points $\xi_{1}=\left(x_{1}, P_{\varepsilon}\left(x_{1}\right)\right), \xi_{2}=\left(x_{2}, P_{\varepsilon}\left(x_{2}\right)\right)$ on the graph of the Poincaré map and put (see Lemma 5.6)

$$
\begin{array}{ll}
z^{1}:=z\left(x_{1}\right), & z^{2}:=z\left(x_{2}\right), \\
z_{n}^{1}:=z_{n}\left(x_{1}\right), & z_{n}^{2}:=z_{n}\left(x_{2}\right) .
\end{array}
$$

We assume without loss of generality that $x_{2}>x_{1}$. By Lemma 5.5 ,

$$
\begin{aligned}
\Delta\left(\xi_{1}\right)-\Delta\left(\xi_{2}\right)= & P_{\varepsilon}\left(x_{1}\right)-x_{1}-\left(P_{\varepsilon}\left(x_{2}\right)-x_{2}\right) \\
= & x_{2}-x_{1}-\left(P_{\varepsilon}\left(x_{2}\right)-P_{\varepsilon}\left(x_{1}\right)\right) \\
= & O^{*}\left(\exp \left(\frac{-I^{+}\left(z^{1}, z^{2}\right)+O\left(\varepsilon^{\lambda}\right)}{\varepsilon}\right)\right) \\
& \quad-O^{*}\left(\exp \left(\frac{I^{-}\left(z^{1}, z^{2}\right)+O\left(\varepsilon^{\lambda}\right)}{\varepsilon}\right)\right) \\
= & O^{*}\left(\exp \left(\frac{-I^{+}\left(z_{n}^{1}, z_{n}^{2}\right)+O\left(\varepsilon^{\lambda}\right)}{\varepsilon}\right)\right) \\
& \quad-O^{*}\left(\exp \left(\frac{I^{-}\left(z_{n}^{1}, z_{n}^{2}\right)+O\left(\varepsilon^{\lambda}\right)}{\varepsilon}\right)\right),
\end{aligned}
$$

where the last equation holds by (5.14).

Hence the sign of $\Delta\left(\xi_{1}\right)-\Delta\left(\xi_{2}\right)$ is determined by the sign of $I^{-}\left(z_{n}^{1}, z_{n}^{2}\right)+I^{+}\left(z_{n}^{1}, z_{n}^{2}\right)$. Thus part 5 holds if we impose finitely many additional nondegeneracy conditions on the system:

$$
I^{-}\left(z_{n}^{i}, z_{n}^{j}\right)+I^{+}\left(z_{n}^{i}, z_{n}^{j}\right) \neq 0, \quad i \neq j
$$

for all pairs of neutral points $z_{i} \neq z_{j}$.

Part 6 is proved in a similar fashion. Consider an interval $J^{+}:=[p, q\rangle$ intersecting the unstable part of the slow curve $M$ near the jump point $G^{+}$. Consider the trajectory passing through its lowest point $p$. We point out that the contour $Z^{+}(p)$ consists of one arc of the unstable part of the slow curve, which can be made arbitrarily small by the choice of $J^{+}$. We also consider an arbitrary neutral point $\xi$ and set $z_{n}=z_{n}(\xi)$. Similarly to (5.16) we have

$$
\begin{aligned}
\Delta\left(A_{\varepsilon}^{-}\right)-\Delta(\xi)=O^{*} & \left(\exp \left(\frac{-I^{+}\left(p, z_{n}\right)+O\left(\varepsilon^{\lambda}\right)}{\varepsilon}\right)\right) \\
& -O^{*}\left(\exp \left(\frac{I^{-}\left(p, z_{n}\right)+O\left(\varepsilon^{\lambda}\right)}{\varepsilon}\right)\right),
\end{aligned}
$$

and the second summand dominates, since the exponent of the exponential in the first summand can be made arbitrarily small.

The assertion for the second endpoint $q$ of the segment $J^{+}$is proved similarly. Part 6 is proved. This completes the proof of all the parts of Lemma 3.5, except for part 2. We shall prove this in the next subsection.

5.4. An upper bound for the number of neutral contours. To estimate the number of neutral contours, that is, the number of zeros of the function $\Phi(z)$, we need to investigate the global continuity of this function on its entire domain of definition. As 
we observed in the preceding subsection, $\Phi(z)$ is continuous on the edges of the graph $\mathcal{G}$. We would like to extend it to a continuous function on the entire domain of definition.

First we return to the simple example we looked at in $\$ 5.1$. It is easy to see that when the initial point $x$ goes right around a vertical circle, the canard jump point $z(x)$ of the corresponding trajectory goes all around the graph $\mathcal{G}$ passing over every edge once but visiting each of the jump points (apart for the principal ones) twice. The function $\Phi(z)$ varies continuously as the jump point goes around, but in general it can take different limit values at the same jump point. Thus, in order to be able to work with $\Phi(z)$ as with a continuous function, we have to 'unglue' the graph $\mathcal{G}$ into a circle which accords with the way the jump point goes around. This circle is the natural domain of definition of the function $\Phi(z)$, and the function is continuous on it. This observation motivates the following construction.

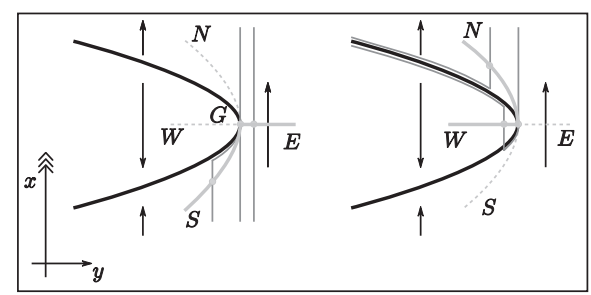

Figure 10. Contours close to a through limit contour of a jump point $G$ (on the left) and a support limit contour (on the right).

It is easy to see that all the jump points, except for the principal ones, belong to one of the four classes depending on the jump type (forward/reverse) and the direction of motion after the jump (upwards/downwards); see Figure 11. Consider the jump point $G$ depicted in Figure 10 (this is one of the four possible configurations; the others are considered in a similar way; see below) and the part of the graph $\mathcal{G}$ situated in a neighbourhood of this point. We denote the edges of the graph entering $G$ by $N, W, S, E$, as shown in the picture.

As the point $z$ approaches the jump $G$ along the edge $E$, the limit position of the corresponding contour $Z(z)$ does not contain arcs of the slow curve in a neighbourhood of $G$. The contour $Z(z)$ tends to the same limit position as $z$ approaches $G$ along the edge $S$. We call such a limit contour a through contour of the point $G$, and the pair of edges $E-S$ a through pair (in the picture on the left).

By contrast, when $z$ tends to $G$ along the edge $N$, the limit position of the contour $Z(z)$ contains the whole unstable arc entering $G$. The contour $Z(z)$ tends to the same position as $z$ moves along the edge $W$. We call such a limit contour a support contour for the point $G$, and the pair of edges $N-W$ a support pair (in the picture on the right).

It is easy to show that the situation is similar for the other types of jump points: the edges of the graph $\mathcal{G}$ entering a jump point are divided into two pairs, and the corresponding contour has the same limit position for each pair. Figure 11 shows pairs of edges for all four configurations: if both edges in a pair lie on one side of the vertical line passing through a jump point, then it is a support pair; otherwise it is a through pair. As the point $z$ goes around the graph in the natural way described at the beginning of the subsection, it enters a vertex along one of the paired edges and exits along the other.

We now modify the graph $\mathcal{G}$ as follows. We replace every vertex $G$ that is not a principal jump point by a pair of vertices $G_{t}$ and $G_{p}$. We assume that the vertex $G_{t}$ is connected with the through pair of edges entering $G$ (we call it a through vertex), and 


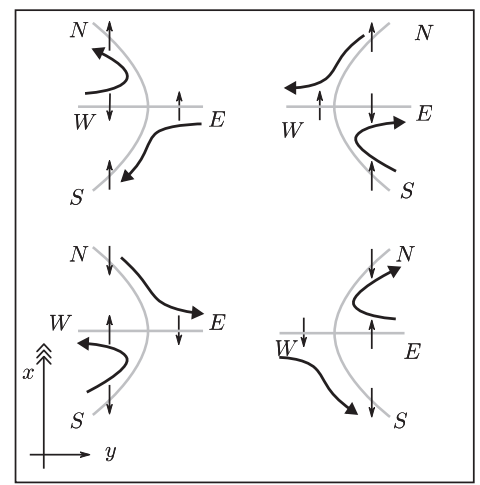

FiguRE 11. Through and support pairs of edges for various types of jump points.

the vertex $G_{p}$ with the support pair of edges (a support vertex). We denote the resulting graph by $\widetilde{\mathcal{G}}$.

Extending the function $\Phi(z)$ to the points $G_{t}$ and $G_{p}$ by continuity we obtain a function that is continuous on the entire graph $\widetilde{\mathcal{G}}$. It can be shown that the resulting graph $\widetilde{\mathcal{G}}$ is connected and so it is a topological circle, but we will not prove this here, as we do not need it for the proof.

It is easy to see that the function $\Phi(z)$, which is monotonic on the edges of $\widetilde{\mathcal{G}}$, is also monotonic at the through vertices of $\widetilde{\mathcal{G}}$ (since $\Phi(z)$ increases monotonically as $y(z)$ increases) and has local extrema at the support points, as well as at the principal jump points. Since the total number of support points and principal jump points is equal to the total number of jump points of the original system and the function $\Phi(z)$ is monotonic between any two support points, the number of zeros of $\Phi(z)$ is bounded by the number of jump points, which proves part 2 of Lemma [3.5, and thus also Theorem 2.5.

We note that the balancing conditions in the statement of Theorem 2.7 can be reformulated as follows:

1. The integral of the support contour corresponding to the jump point $E$ (see Figure 1) is positive.

2. The integral of the support contour corresponding to the jump point $D$ is negative.

Since the integral of the support contour corresponding to $G^{+}$is always negative (this contour contains only stable parts of the slow curve), and the integral of the support contour corresponding to $G^{-}$is always positive, in this case the function $\Phi$ has 4 extremum points with alternating signs, and therefore it has exactly 4 zeros. This proves Lemma 3.6, and thus also Theorem 2.7.

5.5. Basins of attraction. In this subsection we discuss and prove Proposition 2.6.

It follows from Lemma 3.1 that the fixed points of the Poincaré map corresponding to canard cycles lie on an exponentially narrow interval. The 'basin of attraction' of an attracting point (on the transversal $\Gamma$ ) is the interval between two repelling points neighbouring this attracting point. Thus, every attracting point, apart from one, has a basin of attraction with exponentially small measure (a similar assertion can be made for repelling points and their basins of repulsion).

The situation is different on the whole torus: the measure of basins of attraction (repulsion) of the corresponding canard cycles on the whole torus is separated from 
zero. Indeed, for every $\varepsilon$, the neutral trajectories divide the phase space into disjoint domains. At most one canard cycle may be situated between two consecutive neutral jumps. Furthermore, the neutral jumps themselves pass near different neutral contours, and the measure of the domains between them is separated from zero. This implies that any attracting canard cycle has at least one 'neighbouring' repelling cycle such that the measure of the domain between them (contained in the basin of attraction) is separated from zero.

This argument proves Proposition 2.6.

\section{The MAXimal NUMBER OF CANARD CYCLES}

In this section we prove Theorem 2.2 on the existence of an open set of systems having the maximal number of canard solutions.

(a)

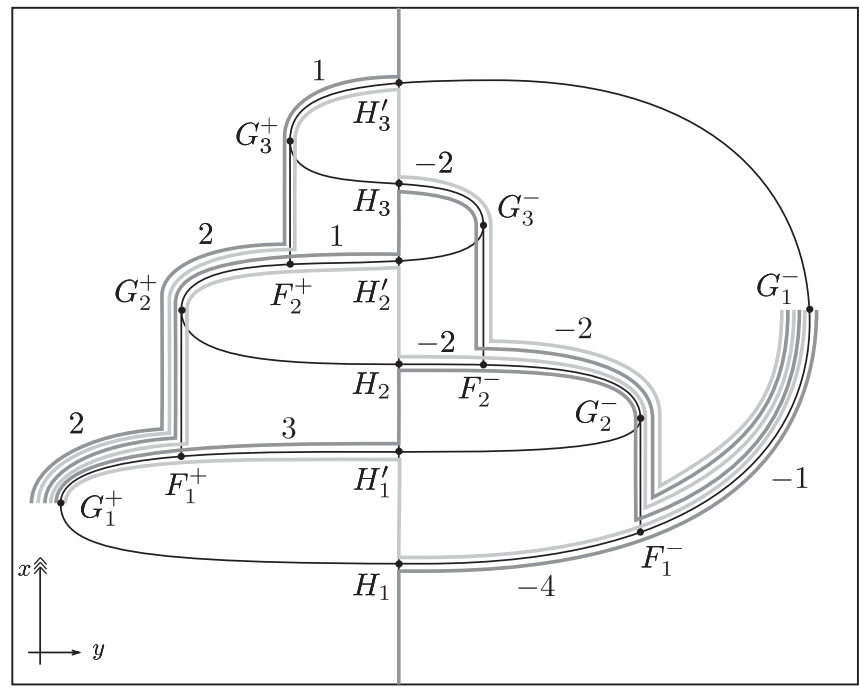

(b)

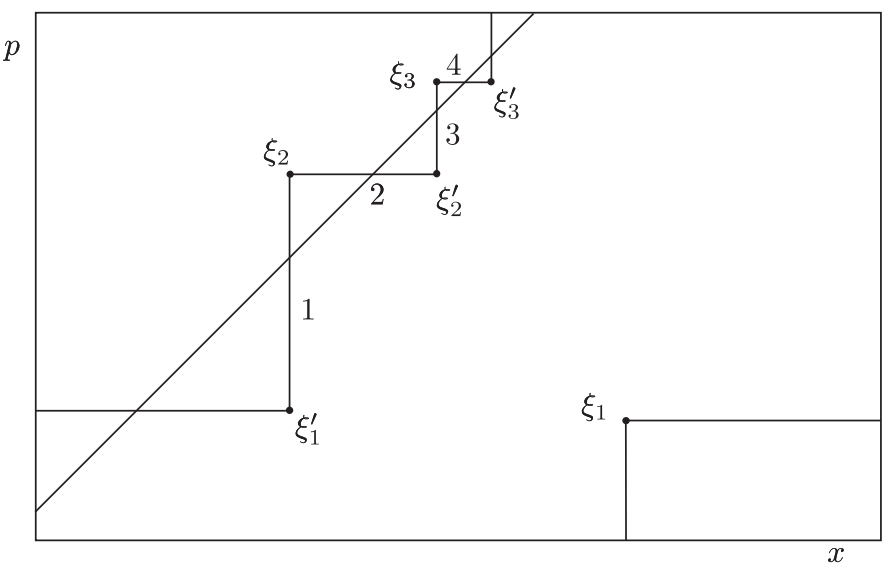

FiguRE 12. (a) A system with a maximal number of canard solutions for $N=3$ and (b) an approximate graph of the Poincaré map (a fragment is enlarged).

Recall that to every neutral contour there corresponds a neutral point on the graph of the Poincaré map such that when it crosses the diagonal $\mathcal{D}$ a pair of canard cycles is 
born or destroyed (see §3.4). Thus the total number of canard solutions does not exceed the total number of neutral points, and it is maximal in the case where firstly $N$ births happen consecutively ( $\mathcal{D}$ is crossed by neutral points at which the graph is convex up), and then $N$ destructions (at the corresponding points the graph is convex down). In other words, this means that the necessary and sufficient conditions for the existence of a maximal number of canard cycles are:

1. The number of neutral contours, $2 K$, is maximal and is equal to $2 N$, the number of folds of the slow curve.

2. If $S_{\Delta}^{1}$ is the circle onto which the torus $\mathbb{T}^{2} \supset \gamma_{\varepsilon}$ is projected by the map $\Delta$, then $S_{\Delta}^{1}$ is divided into the union of two disjoint arcs, one of which contains the images of all the neutral points that 'give birth', and the other contains the images of all the 'destroying' neutral points; this condition is equivalent to the fact that during the motion along the circle there are $N$ births, and then $N$ destructions, of pairs of canard limit cycles.

The first condition is equivalent to the presence of $2 N$ zeros of the function $\Phi(z)$ on the graph $\mathcal{G}$, which in turn is equivalent to the fact that the sign of $\Phi(z)$ alternates at the support vertices of the graph $\widetilde{\mathcal{G}}$ (see $\$ 5.4$ ). In other words, this condition is given by a system of strict inequalities. By part 5 of Lemma 3.5 and its proof (see p. 193), the second condition is also equivalent to a system of strict inequalities on the integrals $\Phi$ over some $\operatorname{arcs}$ of $M$.

Hence the set of systems that have the maximal number of canard solutions is open (as a solution of a system of strict inequalities). It remains to prove that it is nonempty. To do this, for every $N$ it is sufficient to construct a concrete example of a system that has the required property. The rest of this section is devoted to this construction.

It is easy to see that for condition 2 to hold it is sufficient that all the neutral points, apart from one, lie on one exponentially narrow arc and the 'height' and 'width' of the 'steps' on the graph of the Poincare map diminish consecutively: the width of a step is less than its height, and the height of the next step is less than the width of the preceding one; furthermore, the segments between two consecutive neutral points tend to vertical/horizontal ones (see Figure 12 (b)). In this case the diagonal with slope 1 passing through the 'highest' step (in the picture, this is the point $\xi_{3}^{\prime}$ ) passes over all the 'birth-giving' neutral points and under all the 'destroying' ones, since the location (over or under the diagonal) of every next neutral point is determined by the sum of an alternating series in which every successive term is greater in absolute value than the preceding one. The sum of the terms of such a series also alternates.

We construct a system for which the graph of the Poincaré map has this property.

Consider a system that has $2 N$ folds. We label them in order as we go around the slow curve $M$ 'clockwise' by $G_{1}^{-}$(a principal jump point), $G_{1}^{+}$(a principal reverse jump point), $G_{2}^{-}, G_{2}^{+}, \ldots, G_{N}^{-}, G_{N}^{+}$. (We have adopted a new notation because it works better in this situation than the notation in $\$ 5.2$.)

We require that the folds be situated so that the following relations hold (see Figure 12 (a)):

$$
y\left(G_{1}^{+}\right)<y\left(G_{2}^{+}\right)<\cdots<y\left(G_{N}^{+}\right)<y\left(G_{N}^{-}\right)<\cdots<y\left(G_{2}^{-}\right)<y\left(G_{1}^{-}\right),
$$

where all the inequalities are imposed on the coordinates of points on the fundamental square, which contains the whole of $M$.

We construct some vertical circle $\Gamma^{\prime}$ lying between $G_{N}^{+}$and $G_{N}^{-}$. We denote the intersection points of $\Gamma^{\prime}$ with the slow curve $M$ as follows (listed from the bottom up): $H_{1}$, $H_{1}^{\prime}, \ldots, H_{N}, H_{N}^{\prime}$. We denote the contour of any point situated on the interval $\left(H_{i}, H_{i}^{\prime}\right)$ by $Z_{i}^{\prime}$, and the contour of any point on the interval $\left(H_{i}^{\prime}, H_{i+1}\right)$ by $Z_{i+1}$ (see Figure 12 (a)). 
We require that all these contours be neutral. We denote the corresponding neutral points of the graph of the Poincaré map by $\xi_{i}$ and $\xi_{i}^{\prime}$. To avoid confusion, the second coordinate of points $\xi \in \gamma_{\varepsilon}$ is denoted by $p$ (thus, $\xi \equiv(x(\xi), p(\xi))$ ).

For every jump point $G_{i}^{ \pm}, i=2, \ldots, N$, we consider the corresponding fall point and denote it by $F_{i-1}^{ \pm}$.

It follows from Lemma [5.5 that the 'height of the step' $\xi_{i+1}$ is an exponentially small quantity, and the exponent of the exponential is proportional to the integral $\Phi\left(\left(Z_{i}^{\prime}\right)^{-} \cap Z_{i+1}^{-}\right)$(recall that the upper index "+" ("-") of a contour $Z$ means the left (right) half of the contour with respect to a canard jump point $w$; see Definition 5.1 and (5.1)):

$$
h_{i}:=p\left(\xi_{i+1}\right)-p\left(\xi_{i}\right)=O^{*}\left(\exp \left(\frac{-\widetilde{h}_{i}+o(1)}{\varepsilon}\right)\right), \quad i=1, \ldots, N-1,
$$

where $\widetilde{h}_{i}:=-\Phi\left(\left(Z_{i}^{\prime}\right)^{-} \cap Z_{i+1}^{-}\right)>0$. Similarly, for the 'width' of the steps:

$$
w_{i}:=x\left(\xi_{i}^{\prime}\right)-x\left(\xi_{i}\right)=O^{*}\left(\exp \left(\frac{-\widetilde{w}_{i}+o(1)}{\varepsilon}\right)\right), \quad i=2, \ldots, N,
$$

where $\widetilde{w}_{i}:=\Phi\left(\left(Z_{i}^{\prime}\right)^{+} \cap Z_{i}^{+}\right)>0$. For the required relations to hold it is sufficient that the following is satisfied:

$$
h_{1}>w_{2}>h_{2}>\cdots>h_{N-1}>w_{N}
$$

or, equivalently, for a sufficiently small $\varepsilon$ :

$$
\widetilde{h}_{1}<\widetilde{w}_{2}<\widetilde{h}_{2}<\cdots<\widetilde{h}_{N-1}<\widetilde{w}_{N} .
$$

We claim that we can ensure that this relation holds by choosing the values of the integrals $\Phi$ over arcs of $M$ suitably. To do this we set

$$
\begin{aligned}
& \Phi\left(F_{1}^{-} G_{1}^{-}\right)=-1 ; \\
& \Phi\left(G_{i}^{+} F_{i}^{+}\right)=2, \quad i=1, \ldots, N-1 ; \\
& \Phi\left(F_{i}^{-} G_{i}^{-}\right)=-2, \quad i=2, \ldots, N-1 .
\end{aligned}
$$

It is easy to see that

$$
\begin{aligned}
\left(Z_{i}^{\prime}\right)^{-} \cap Z_{i+1}^{-} & =\bigcup_{j=1}^{i}\left(F_{j}^{-} G_{j}^{-}\right), \quad i=1, \ldots, N-1, \\
\left(Z_{i}^{\prime}\right)^{+} \cap Z_{i}^{+} & =\bigcup_{j=1}^{i-1}\left(G_{j}^{+} F_{j}^{+}\right), \quad i=2, \ldots, N,
\end{aligned}
$$

whence

$$
\widetilde{h}_{i}=2 i-1, \quad \widetilde{w}_{i}=2 i-2,
$$

and (6.5) follows immediately.

It remains to show that the contours $Z_{i}$ and $Z_{i}^{\prime}$ can be made neutral. In addition, we set:

$$
\begin{aligned}
\Phi\left(H_{N} G_{N}^{-}\right) & =-2 ; \\
\Phi\left(G_{N}^{+} H_{N}^{\prime}\right) & =1 ; \\
\Phi\left(H_{i} F_{i}^{-}\right) & =-(2 N-2 i), \quad i=1, \ldots, N-1 ; \\
\Phi\left(F_{i}^{+} H_{i}^{\prime}\right) & =2 N-2 i-1, \quad i=1, \ldots, N-1 .
\end{aligned}
$$


Consider the contour $Z_{i}^{\prime}, i=1, \ldots, N-1$ :

$$
Z_{i}^{\prime}=\bigcup_{j=1}^{i}\left(G_{j}^{+} F_{j}^{+}\right) \cup\left(F_{i}^{+} H_{i}^{\prime}\right) \cup\left(H_{i} F_{i}^{-}\right) \cup \bigcup_{j=1}^{i}\left(F_{j}^{-} G_{j}^{-}\right) .
$$

Thus,

$$
\begin{aligned}
\Phi\left(Z_{i}^{\prime}\right) & =\widetilde{w}_{i+1}+\Phi\left(F_{i}^{+} H_{i}^{\prime}\right)+\Phi\left(H_{i} F_{i}^{-}\right)-\widetilde{h}_{i} \\
& =2 i+2 N-2 i-1-(2 N-2 i)-(2 i-1) \\
& =0 .
\end{aligned}
$$

Similarly, we can show that $\Phi\left(Z_{i}\right)=0$ for $i=1, \ldots, N-1$. A similar direct verification also shows that $\Phi\left(Z_{N}\right)=0$ and $\Phi\left(Z_{N}^{\prime}\right)=0$.

The slope of the segments $\left[\xi_{i}^{\prime}, \xi_{i+1}\right]$ tends to infinity, since the distance $x\left(\xi_{i+1}\right)-x\left(\xi_{i}^{\prime}\right)$ is exponentially small compared to $p\left(\xi_{i+1}\right)-p\left(\xi_{i}^{\prime}\right)$ (this is easy to see if we apply Lemma 5.5] to the corresponding half-contours). Similarly it can be shown that the slope of the segments $\left[\xi_{i}, \xi_{i}^{\prime}\right]$ tends to zero.

The theorem is proved.

\section{NONLINEAR EFFECTS}

In this section we prove the technical lemmas stated in $\$ 5.2$.

\subsection{Singular trajectories.}

Proof of Lemma 5.3. We only need to prove the assertion of the lemma corresponding to forward time. The proof for reverse time is carried out by applying the same arguments to the system with time reversed.

We consider the contour $Z^{-}(w)$ and add the vertical segments connecting $w$ with $F^{-}(w)$ to it and also the vertical segments connecting the jump points $g$ with the corresponding fall points $F^{-}(g)$. We obtain a continuous curve, which starts at the point $w$, and ends at the principal jump point $G^{-}$; it is entirely contained inside the domain $\Sigma_{\delta}$ for any $\delta>0$. This curve is called a singular trajectory of the system; we denote it by $\widehat{Z}^{-}(w)$. It is well known (see [9]) that under the nondegeneracy conditions listed in $\$ 2$ the true trajectory of the fast-slow system passing through the point $w$ tends uniformly to the corresponding singular trajectory as $\varepsilon \rightarrow 0$ (this can be shown by explicitly constructing an arbitrarily small capturing neighbourhood of the singular trajectory). Thus, moving near the singular trajectory, for sufficiently small $\varepsilon$, the trajectory cannot leave the neighbourhood $\Sigma_{\delta / 2}$ until it exits the strip $\Pi$.

7.2. Exponential contraction. In what follows we need two classical theorems describing the behaviour of the true slow surface near a jump point, which we state here.

Theorem 7.1 (9]). Suppose that a system of the form (2.1) has a nondegenerate jump point at the origin. There exists a small $r>0$ such that for every $\mu \in(0,1 / 3)$ the true slow curve $x=s(y, \varepsilon)$ over the segment $\left[-r,-\varepsilon^{\mu}\right]$ satisfies the following equation:

$$
s(y, \varepsilon)=s(y)+O\left(\varepsilon^{2 / 3-\mu / 2}\right) .
$$

To derive this theorem from formula (16.10) in the given reference, it is enough to make a trivial change of coordinates.

Theorem 7.2 (ibid., see also [4]). Under the hypotheses of the preceding theorem the continuation of the true slow curve $s(y, \varepsilon)$ beyond the jump point intersects a horizontal section $x=-$ const at a point with coordinate $y=O\left(\varepsilon^{2 / 3}\right)$. 
Lemma 7.3. Consider some vertical segment

$$
J=\left\{(x, y) \mid x \in I, y=y^{*}\right\},
$$

intersecting a stable part of the slow curve $M$ and not intersecting the $\delta$-neighbourhood of any unstable point of $M$ (in particular, $J$ is separated from jump points). The contour $Z^{-}=Z^{-}(w)$ is obviously independent of the choice of the point $w \in J$ for fixed $J$. Then for some $\lambda>0$ the following representation holds for the derivative of the Poincaré map:

$$
\left.\ln \frac{d}{d x} P_{\varepsilon}^{\left[y^{*}, \pi\right\rangle}\right|_{x=x^{*}}=\frac{\Phi^{-}\left(Z^{-}\right)+O_{\delta}\left(\varepsilon^{\lambda}\right)}{\varepsilon}, \quad x^{*} \in I,
$$

where the remainder term $O_{\delta}\left(\varepsilon^{\lambda}\right)$ is bounded by $C_{\delta} \varepsilon^{\lambda}$, where $C_{\delta}$ depends only on $\delta$ and is independent of the choice of the interval $J$ and the point $x^{*}$.

Proof. We fix $w$ and consider the trajectory $y=y(x ; w, \varepsilon)$ passing through $w$. We fix some sufficiently small $\delta^{\prime}>0$.

Next, for every arc of the slow curve $\left[F_{j}, G_{j}\right\rangle$ contained in the contour $Z^{-}$we define several vertical circles that are given by their $y$-coordinate: $\Gamma_{j}^{i}=\left\{y=y_{j}^{i}\right\}$ (see Figure13, some of these circles will depend on $\varepsilon$ ), where

$$
\begin{aligned}
& y_{j}^{0}:=y\left(F_{j}\right), \\
& y_{j}^{3}:=y\left(G_{j}\right)-\delta^{\prime}, \\
& y_{j}^{4}:=y\left(G_{j}\right)-\varepsilon^{1 / 4} .
\end{aligned}
$$

We set $A_{j}^{i}:=\left[F_{j}, G_{j}\right\rangle \cap \Gamma_{j}^{i}$. By Theorem 4.2, the normal form (4.4) is in force near the arc $\left[F_{j}, A_{j}^{3}\right\rangle$. We define the following domain $U_{j}$ :

$$
U_{j}:=\left\{(x, y)\left|y \in\left[y_{j}^{0}, y_{j}^{3}\right\rangle,\right| \widetilde{x} \mid<b\right\},
$$

where $\widetilde{x}$ is the normalizing coordinate in the corresponding domain.

Suppose that the trajectory enters the neighbourhood $U_{j}$ at a point $B_{j}$ (that is, $\left|\widetilde{x}\left(B_{j}\right)\right|=b$; if $j=1$ and the point $w$ is already contained in the domain $U_{1}$, then we assume $\left.B_{1}=w\right)$. We set $y_{j}^{2}:=y\left(B_{j}\right)$.

(a)

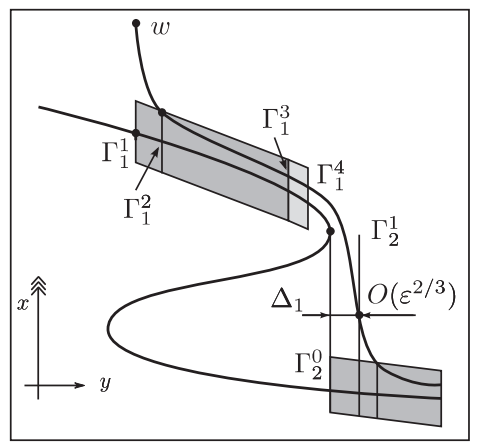

(b)

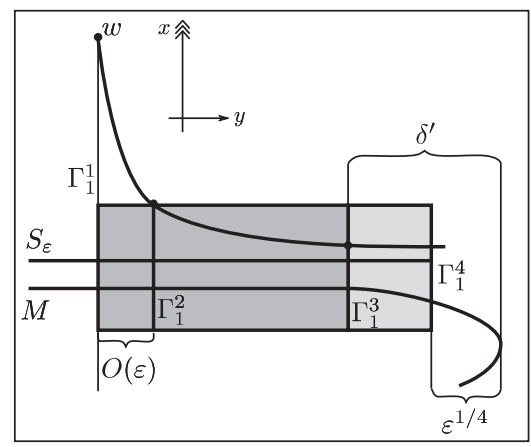

Figure 13. (a) Part of the trajectory; (b) enlarged fragment of the domain $U_{1}$ in normalized coordinates.

For every jump $G_{j}$ that occurs in the contour $Z^{-}(w)$ but is not the principal jump $G^{-}$, we fix some horizontal segment $\Delta_{j}$ intersecting the jump interval $\left(G_{j} F_{j+1}\right)$ (of the two vertical arcs with endpoints at these points, we choose the one that does not intersect $M$ at its inner points) that is at a distance of at least $\delta$ from points of the slow curve $M$. Suppose that the trajectory intersects $\Delta_{j}$ at a point $E_{j}$ (we assume that $E_{0}:=w$ ). We set $y_{j}^{1}:=y\left(E_{j}\right)$. 
We represent the Poincaré map $P_{\varepsilon}^{\left[y^{*}, \pi\right\rangle}$ as the composite

$$
P_{\varepsilon}^{\left[y^{*}, \pi\right\rangle}: \Gamma_{1}^{1} \stackrel{P^{1}}{\rightarrow} \Gamma_{2}^{1} \stackrel{P^{2}}{\rightarrow} \cdots \stackrel{P^{m-1}}{\rightarrow} \Gamma_{m}^{1} \stackrel{P^{m}}{\rightarrow} \Gamma
$$

where $P_{j}=P_{\varepsilon}^{\left[y_{j}^{1}, y_{j+1}^{1}\right\rangle}$ and $y_{m+1}^{1}:=\pi$. We now prove that for every $j=1, \ldots, m$ and for some $\lambda>0$ we have the representation

$$
\left.\ln \frac{d}{d x} P_{j}\right|_{x=x\left(y_{j}^{1} ; w, \varepsilon\right)}=\frac{\Phi\left(\left[F_{j}, G_{j}\right\rangle\right)+O\left(\varepsilon^{\lambda}\right)}{\varepsilon} .
$$

When taking the derivative in (7.8), the decomposition (7.7) is regarded as fixed. To prove (7.8) we represent each of the maps $P_{j}$ in turn in the form of a composite:

$$
\begin{gathered}
P_{j}: \Gamma_{j}^{1} \stackrel{P_{j}^{1}}{\rightarrow} \Gamma_{j}^{2} \stackrel{P_{j}^{2}}{\rightarrow} \Gamma_{j}^{3} \stackrel{P_{3}^{4}}{\rightarrow} \Gamma_{j}^{4} \stackrel{P_{i}^{5}}{\rightarrow} \Gamma_{j+1}^{1}, \\
P_{m}: \Gamma_{m}^{1} \stackrel{P_{m}^{1}}{\rightarrow} \Gamma_{m}^{2} \stackrel{P_{m}^{2}}{\rightarrow} \Gamma_{m}^{3} \stackrel{\widetilde{P}_{m}^{3}}{\rightarrow} \Gamma,
\end{gathered}
$$

where $j=1, \ldots, m-1$.

Next we estimate the logarithmic derivative of each of the maps $P_{j}^{i}=P_{\varepsilon}^{\left[y_{j}^{i}, y_{j}^{i+1}\right\rangle}$.

Since the point $E_{j}$ lies outside a neighbourhood of the unstable part of the slow curve, from $E_{j}$ the trajectory of the system enters the fixed neighbourhood $U_{j+1}$ of the stable part of the slow curve in a time that is bounded above. This implies that

$$
y_{j}^{2}-y_{j}^{1}=O(\varepsilon)
$$

and Lemma 4.5 gives

$$
\left|\ln \frac{d}{d x} P_{j}^{1}\right|<C
$$

for some universal constant $C$ independent of $w$.

In view of the normal form (4.4), in a suitable system of coordinates the map $P_{j}^{2}$ is linear, and its derivatives can be calculated by integrating the normal form explicitly. We have

$$
\ln \frac{d}{d x} P_{j}^{2}=\frac{\Phi\left(\left[A_{j}^{2}, A_{j}^{3}\right\rangle\right)}{\varepsilon} .
$$

It follows from Theorem 7.2 that $y_{j}^{1}-y_{j}^{0}=O\left(\varepsilon^{2 / 3}\right)$. Together with (7.11) we also have $y_{j}^{2}-y_{j}^{0}=O\left(\varepsilon^{2 / 3}\right)$. Because the integral is smooth with respect to the integration limits, this implies that we have the representation $\Phi\left(\left[A_{j}^{2}, A_{j}^{3}\right\rangle\right)=\Phi\left(\left[F_{j}, A_{j}^{3}\right\rangle\right)+O\left(\varepsilon^{2 / 3}\right)$, where the estimate for the remainder term is independent of the choice of the point $w$.

We observe that the map $P_{j}^{2}$ is an exponential contraction that can be made arbitrarily strong by comparison with a possible expansion on the interval $\Gamma_{j}^{3} \rightarrow \Gamma_{j}^{4}$ by choosing a sufficiently small $\delta^{\prime}$ (this follows, in particular, from the estimate of Lemma 4.5). This means that the trajectory intersects $\Gamma_{j}^{3}$ at a point that is exponentially close to the intersection point of the true slow curve with $\Gamma_{j}^{3}$, after which the trajectory remains exponentially close to the true slow curve as it proceeds along the entire interval $\Gamma_{j}^{3} \rightarrow \Gamma_{j}^{4}$. From the variational equation, in the notation of Theorem [7.1], we have

$$
\begin{aligned}
\ln \frac{d}{d x}\left(P_{j}^{4}\right) & =\int_{y_{j}^{3}}^{y_{j}^{4}} f_{x}^{\prime}(x(y), y, \varepsilon) d y=\int_{y_{j}^{3}}^{y_{j}^{4}} f_{x}^{\prime}(s(y, 0), y, \varepsilon) d x+O(\varepsilon) \\
& =\int_{y_{j}^{3}}^{y_{j}^{4}} f_{x}^{\prime}(s(y), y, 0)+O\left(\varepsilon^{2 / 3-\mu / 2}\right)=\int_{y_{j}^{3}}^{y_{j+1}^{0}} f_{x}^{\prime}(s(y), y, 0)+O\left(\varepsilon^{1 / 4}\right) \\
& =\Phi\left(A_{j}^{3}, G_{j}\right)+O\left(\varepsilon^{1 / 4}\right) .
\end{aligned}
$$


From the relation $y_{j+1}^{0}-y_{j}^{4}=O\left(\varepsilon^{1 / 4}\right)$, using Lemma 4.5, the derivative of the map $P_{j}^{5}$ admits the estimate:

$$
\left|\ln \frac{d}{d x} P_{j}^{5}\right|=\frac{O\left(\varepsilon^{1 / 4}\right)}{\varepsilon} .
$$

The derivative of the map $\widetilde{P}_{m}^{3}$ is estimated by using Theorem 4.6

$$
\ln \frac{d}{d x} \widetilde{P}_{m}^{3}=\frac{\Phi\left(A_{m}^{3} G^{+}\right)+O\left(\varepsilon^{\lambda}\right)}{\varepsilon}
$$

for some $\lambda>0$.

Using the chain rule and taking the sum of the representations obtained for the logarithmic derivatives of the functions $P_{j}^{i}$, as well as of the function $\widetilde{P}_{m}^{3}$, we obtain the required representation (7.8). The estimate for the remainder term is uniform with respect to the point $w$. The lemma is proved.

Proof of Lemma 5.4. Equation (5.8) follows directly from Lemma 7.3 . Equation (5.9) is proved in a similar fashion, by applying this lemma to the system with reversed time, and equation (5.10) follows from the two preceding equations using the chain rule.

Proof of Lemma 5.5. It is sufficient to prove relation (5.13). Consider the left-most point $F_{j_{0}}$ of the intersection of the contours $Z^{-}\left(w_{1}\right) \cap Z^{-}\left(w_{2}\right)$. By the nondegeneracy condition (2.3), one of the contours $Z^{-}\left(w_{1}\right), Z^{-}\left(w_{2}\right)$ contains an arc which contains the point $F_{j_{0}}$ together with some neighbourhood of the slow curve $M$. We assume without loss of generality that this arc is $\left[F_{k, 2}^{1}, G_{k, 2}^{1}\right\rangle \subset Z^{-}\left(w_{2}\right)$.

Consider an interval $J_{j_{0}}^{2}$ lying on the circle $\Gamma_{j_{0}}^{2}$ for the contour $Z^{-}\left(w_{1}\right)$ (see the proof of Lemma 7.3 in the preceding subsection) and intersecting the stable part of the slow curve. The trajectory $x=x_{1}(y, \varepsilon)$ passing through the point $w_{1}$ intersects this interval at a point with fixed coordinate $b \neq 0$ in the normalizing chart. The trajectory $x=x_{2}(y, \varepsilon)$ passing through the point $w_{2}$ passes near the arc $\left[F_{k, 2}^{1}, G_{k, 2}^{1}\right\rangle$ and up to the moment when it intersects $J_{j_{0}}^{2}$ has time to be exponentially attracted to the true slow curve: $x_{2}\left(y_{j_{0}}^{2}, \varepsilon\right)=O\left(e^{-C / \varepsilon}\right)$. Introducing the notation $I_{\varepsilon}:=\left[x_{1}\left(y_{j_{0}}^{2}, \varepsilon\right), x_{2}\left(y_{j_{0}}^{2}, \varepsilon\right)\right] \subset J_{j_{0}}^{2}$ we obtain the estimate

$$
\left|I_{\varepsilon}\right|=b+O\left(e^{-C / \varepsilon}\right) .
$$

Applying Lemma 7.3 to the map $P_{\varepsilon}^{\left[y_{j_{0}}^{2}, \pi\right\rangle}$ on the interval $J_{j_{0}}^{2}$ we obtain a uniform estimate for the derivative, and from Lagrange's theorem on linear increments we obtain the required estimate for the length of the image $P_{\varepsilon}^{\left[y_{j_{0}}^{2}, \pi\right\rangle}\left(I_{\varepsilon}\right)$.

\subsection{Neutral trajectories and neutral contours.}

Proof of Lemma 5.6. Recall that under the hypotheses of the lemma, $x \in U$, that is, $P_{\varepsilon}^{\prime}(x) \in[1 / 2,2]$. Suppose that the value $z(x)$ is not defined; that is, the corresponding trajectory does not leave the domain $\Sigma_{\delta}$ in any way other than through the boundary of the strip $\Pi$. In this case the trajectory passes near some arcs of the stable and the unstable parts of the slow curve, and the switching between arcs is a result of forward and reverse jumps, or a canard jump passing near fold points of the slow curve. It is easy to see that the trajectory can pass from the unstable part of the slow curve to the stable part only once and then it will move near to the stable part. Furthermore, the trajectory passes near the limit contour $Z_{0}$ (a support contour if there are no canard jumps, and a through contour otherwise).

We represent $\Sigma_{\delta}$ as the union

$$
\Sigma_{\delta}=M_{\delta} \cup V_{\delta},
$$


where $M_{\delta}$ denotes the $\delta$-neighbourhood of the slow curve $M$, and $V_{\delta}$ the union of the $\delta$-neighbourhoods of the vertical segments passing through the jump points (see (5.5)). When moving in the domain $M_{\delta} \backslash V_{\delta}$, the trajectory passes a distance at most $\delta$ from the arcs contained in the contour $Z_{0}$. Here its dynamics are determined by the normal form (4.4). The time it moves in $V_{\delta}$ is bounded by the quantity $2 N \delta / \varepsilon$, where $2 N$ is the total number of fold points.

By the variational equation, as well as by Theorem 4.6, the derivative of the Poincaré map at the point $x$ has the following representation:

$$
\ln P_{\varepsilon}^{\prime}(x)=\frac{1}{\varepsilon} \int_{\gamma(x)} f_{x}^{\prime}(u(y), y, \varepsilon) d y+o(1 / \varepsilon),
$$

where $\gamma(x)$ is the intersection of the segment of the trajectory $x=u(y)$ which passes through the point $x$ with the strip $\Pi$. Taking account of the arguments we have given above, as well as Lemma 4.5, when we integrate we can replace the curve $\gamma(x)$ by the contour $Z_{0}$ :

$$
\ln P_{\varepsilon}^{\prime}(x)=\frac{\Phi\left(Z_{0}\right)+O(2 N \delta)+o(1)}{\varepsilon} .
$$

Choosing $\delta$ sufficiently small, we can ensure that the first summand in the numerator dominates and the derivative $P_{\varepsilon}^{\prime}$ is exponentially small or large, contrary to the assumption $P_{\varepsilon}^{\prime}(x) \in[1 / 2,2]$. This contradiction proves that the function $z=z(x)$ is defined at all points of $U$.

In this case, relation (5.14) is an immediate consequence of Lemma 5.4 and the condition $\frac{d}{d y} \Phi(z) \neq 0$, where $y=y(z)$, which follows from the nondegeneracy condition $f_{x}^{\prime}(x, y, 0) \neq 0$, which is valid for any point $(x, y) \in M$ that is not a fold point (see (2.1)).

\subsection{Estimating the second derivative.}

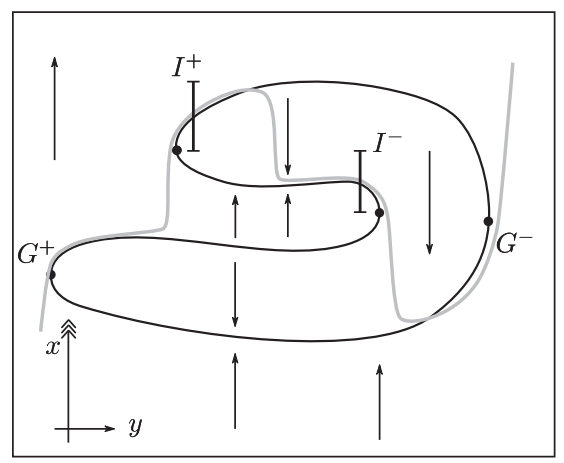

FiguRE 14. Neutral jump.

Proof of Lemma 5.7. Consider the trajectory with initial condition $x \in U$. We set $z=$ $z(x)$ and $z_{n}=z_{n}(x)$ (see Lemma [5.6) and consider the contour $Z=Z\left(z_{n}(x)\right.$ ). Consider the jump points $G_{d}^{ \pm}:=G^{ \pm}(z)$. Let $I^{+}\left(I^{-}\right)$be a vertical segment at distance $\delta$ from the jump point $G_{d}^{+}\left(G_{d}^{-}\right)$that intersects the unstable (stable) part of the slow curve and does not intersect the stable (unstable) part of the slow curve (see Figure 14). We set 
$y^{ \pm}=y\left(I^{ \pm}\right)$. We represent the Poincaré map in a neighbourhood of the point $x$ as the composite

$$
P_{\varepsilon}: \Gamma \stackrel{R}{\rightarrow} I^{+} \stackrel{\widetilde{P}}{\rightarrow} I^{-} \stackrel{Q}{\rightarrow} \Gamma .
$$

It follows from the chain rule that

$$
\begin{aligned}
\ln \frac{d}{d x} P_{\varepsilon}(x) & =\ln Q^{\prime} \circ \widetilde{P} \circ R(x)+\ln \widetilde{P}^{\prime} \circ R(x)+\ln R^{\prime}(x), \\
\frac{d}{d x} \ln \frac{d}{d x} P_{\varepsilon}(x) & =\frac{d}{d x} \ln Q^{\prime} \circ \widetilde{P} \circ R(x)+\frac{d}{d x} \ln \widetilde{P}^{\prime} \circ R(x)+\frac{d}{d x} \ln R^{\prime}(x) .
\end{aligned}
$$

As the logarithm is monotonic, to prove the lemma it is sufficient to determine the sign of (7.23). We will show that in this decomposition the second term dominates and find its sign.

We estimate the third term of the expression (7.23). It follows from the variational equation that

$$
\ln R^{\prime}(x)=\frac{1}{\varepsilon} \int_{-\pi}^{y^{+}} f_{x}^{\prime}(\varphi(y, \varepsilon ; x), y, \varepsilon) d y
$$

where $x=\varphi(y, \varepsilon ; x)$ defines the trajectory with the initial condition $x \in \Gamma$. Differentiating we obtain

$$
\frac{d}{d x} \ln R^{\prime}(x)=\frac{1}{\varepsilon} \int_{-\pi}^{y^{-}} f_{x x}^{\prime \prime}(\varphi(y, \varepsilon ; x), y, \varepsilon) X(y ; x) d y,
$$

where $X(y ; x)=\frac{d}{d x} \varphi(y, \varepsilon ; x)=P_{\varepsilon}^{[-\pi, y\rangle}(x)$.

Let $Z^{+}=Z^{+}\left(z_{n}\right)$ and let $\widehat{G}^{+}$be the set of (reverse) jump points occurring in the contour $Z^{+}$(that is, the set of reverse jump points the trajectory under consideration passes close to). We define a set $W_{\delta}$ as follows:

$$
W_{\delta}=\bigcup_{G \in \widehat{G}^{+}} U_{\delta}(y(G))
$$

where $U_{\delta}(y(G))=[y(G)-\delta, y(G)+\delta\rangle$.

It is easy to see that for $y \notin W_{\delta}$ the point $w(y)=(\varphi(y, \varepsilon ; x), y)$ satisfies the hypotheses of Lemma 7.3 and following from (7.2), for the system with the reversed time, we have the representation:

$$
X(y ; x)=\exp \left(\frac{\Phi\left(Z^{+}(w(y))\right)+O_{\delta}\left(\varepsilon^{\lambda}\right)}{\varepsilon}\right) .
$$

We estimate $X(y ; x)$ for $y \in W_{\delta}$. Let $y \in U_{\delta}\left(y^{*}\right)$, where $y^{*}=y(G)$ for some $G \in \widehat{G}^{+}$. It follows from the chain rule that

$$
X(y ; x)=\frac{d}{d x} P_{\varepsilon}^{\left[-\pi, y^{*}-\delta\right\rangle} \cdot \frac{d}{d x} P_{\varepsilon}^{\left[y^{*}-\delta, y\right\rangle} .
$$

The first factor is estimated by (7.27). The second factor is roughly estimated by using the variational equation (see Lemma 4.5):

$$
\frac{d}{d x} P_{\varepsilon}^{\left[y^{*}-\delta, y\right\rangle}=O\left(e^{O(\delta) / \varepsilon)}\right) .
$$

Thus, for $y \in W_{\delta}$ we have the estimate

$$
X(y ; x)=\exp \left(\frac{\Phi\left(Z^{+}\left(w\left(y^{*}-\delta\right)\right)\right)+O_{\delta}\left(\varepsilon^{\lambda}\right)+O(\delta)}{\varepsilon}\right),
$$

and we can assume that $O_{\delta}\left(\varepsilon^{\lambda}\right)+O(\delta)=O(\delta)$. 
Since $\Phi\left(Z^{+}(w(y))\right)$ increases monotonically with respect to $y$, the right-hand sides of (7.27) and (7.30) do not exceed the quantity

$$
X\left(y^{+} ; x\right)=\exp \left(\frac{\Phi\left(Z^{+}\left(w\left(y^{+}\right)\right)\right)+O(\delta)}{\varepsilon}\right) .
$$

Substituting the estimate we have obtained into the integral (7.25) and taking into account that $\left|f_{x x}^{\prime \prime}\right|<C$, since $f$ is smooth and the phase space is compact, we obtain

$$
\begin{aligned}
\left|\frac{d}{d x} \ln R^{\prime}(x)\right| & <\frac{C}{\varepsilon} \exp \left(\frac{\Phi\left(Z^{+}\left(w\left(y^{+}\right)\right)\right)+O(\delta)}{\varepsilon}\right) \\
& =\exp \left(\frac{\Phi\left(Z^{+}\left(w\left(y^{+}\right)\right)+O(\delta)\right)}{\varepsilon}\right) .
\end{aligned}
$$

By similar arguments, since $\Phi\left(Z^{-}(w(y))\right)$ decreases with respect to $y$, it is easy to obtain the estimate

$$
\frac{d}{d x} \ln Q^{\prime}(x)=\exp \left(\frac{O(\delta)}{\varepsilon}\right)
$$

We denote the part of the contour $Z$ lying on the left of $I^{-}$by $\widetilde{Z}$ :

$$
\widetilde{Z}:=Z \cap\left\{y \in\left[-\pi, y^{-}\right\rangle\right\} \text {. }
$$

Representing the first summand in (7.23) in the form

$$
\frac{d}{d x} \ln Q^{\prime} \circ \widetilde{P} \circ R(x)=\frac{d}{d x}\left(\ln Q^{\prime}(x)\right) \cdot \frac{d}{d x}(\widetilde{P} \circ R(x))
$$

and using Lemma 7.3 to estimate the derivative $\widetilde{P} \circ R(x)=P_{\varepsilon}^{\left[-\pi, y^{-}\right\rangle}$, we obtain

$$
\left|\frac{d}{d x} \ln Q^{\prime} \circ \widetilde{P} \circ R(x)\right|<\exp \left(\frac{\Phi(\widetilde{Z})+O(\delta)}{\varepsilon}\right) .
$$

We estimate the second summand in (17.23) from below. The chain rule and relation (7.27) applied at the point $y=y^{+}$give the representation

$$
\begin{aligned}
\frac{d}{d x} \ln \widetilde{P}^{\prime} \circ R(x) & =\frac{d}{d x} \ln \widetilde{P}^{\prime}(x) \cdot R^{\prime}(x) \\
& =\frac{d}{d x} \ln \widetilde{P}^{\prime}(x) \cdot \exp \left(\frac{\Phi\left(Z^{+}\left(w\left(y^{+}\right)\right)\right)+O\left(\varepsilon^{\lambda}\right)}{\varepsilon}\right) .
\end{aligned}
$$

We now estimate $\frac{d}{d x} \ln \widetilde{P}^{\prime}(x)$. The normal form (4.4) holds near the parts of the slow curve (stable and unstable) between the intervals $I^{ \pm}$. We denote the normalized coordinate near the unstable part of the slow curve by $\xi$, and near the stable part by $\eta$. Let the map $\widetilde{P}: I^{+} \rightarrow I^{-}$be given in the normalizing coordinates as $\xi \mapsto \eta=\eta(\xi)$.

A canard jump at a point $z$ may happen in one of the two directions - upwards or downwards - depending on the sign of $f(z, 0)$. We consider the case where a canard jump happens in the 'downwards' direction (that is, $f(z, 0)<0$; the opposite case is considered in a similar fashion). In this case, $\xi<0$ (that is, the trajectory passes below the corresponding true slow surface and jumps downwards).

Consider the neighbourhoods of the stable and unstable parts of the slow curve that are given by the relations $|\xi|<b$ and $|\eta|<b$ for some fixed $b>0$. Suppose that the trajectory leaves the neighbourhood of the unstable part of the slow curve at the point $\left(\xi=-b, y=y_{0}^{+}\right)$and enters the neighbourhood of the stable part of the slow curve at the point $\left(\eta=b, y=y_{0}^{-}\right)$. Obviously, $y_{0}^{-}=y_{0}^{+}+O(\varepsilon)$. Lemma 5.6 also implies that $y_{0}^{1}=y\left(z_{n}\right)+O\left(\varepsilon^{\lambda}\right)$. Let $L^{+}$be the arc of the unstable part of the slow curve lying between $I^{+}$and $y_{0}^{+}$, and $L^{-}$the arc of the stable part of the slow curve lying between $y_{0}^{-}$ 
and $I^{-}$. Also let $\widetilde{L}^{+}$be the arc of the unstable part of the slow curve lying between $I^{+}$ and $y\left(z_{n}\right)$, and $\widetilde{L}^{-}$the arc of the stable part of the slow curve lying between $y\left(z_{n}\right)$ and $I^{-}$.

As shown in [3] (for the general case, see formula (4.41) in [5]),

$$
\frac{d}{d \xi} \ln \eta^{\prime}(\xi)>-\frac{1}{\xi},
$$

and the normal form (4.4) implies that

$$
-\xi=b \exp \left(\frac{-\Phi\left(L^{+}\right)}{\varepsilon}\right)=b \exp \left(\frac{-\Phi\left(\widetilde{L}^{+}\right)+O\left(\varepsilon^{\lambda}\right)}{\varepsilon}\right) .
$$

Thus, we have the estimate

$$
\frac{d}{d \xi} \eta^{\prime}(\xi)>C \exp \left(\frac{\Phi\left(\widetilde{L}^{+}\right)+O\left(\varepsilon^{\lambda}\right)}{\varepsilon}\right),
$$

where $C=1 / b>0$. The map which passes to the normalizing coordinates has uniformly bounded derivatives with respect to $\varepsilon$ and therefore can only change the constant $C$.

It is easy to see that the contour $Z^{+}\left(w\left(y^{+}\right)\right)$is complemented by the arc $\widetilde{L}^{+}$to the contour $Z^{+}\left(w_{n}\right)$. Thus, by substituting (7.40) into (17.37) we obtain

$$
\frac{d}{d x} \ln \widetilde{P}^{\prime} \circ R(x)>C \exp \left(\frac{\Phi\left(Z^{+}\left(w_{n}\right)\right)+O\left(\varepsilon^{\lambda}\right)}{\varepsilon}\right) .
$$

Obviously, $\Phi\left(Z^{+}\left(w_{n}\right)\right)>\Phi\left(Z^{+}\left(w\left(y^{+}\right)\right)\right)$(since the arc $\widetilde{L}^{+}$of the unstable part of the slow curve was added into the contour on the right-hand side) and $\Phi\left(Z^{+}\left(w_{n}\right)\right)>\Phi(\widetilde{Z})$ (since the arc $\widetilde{L}^{-}$of the stable part of the slow curve was added into the contour on the right-hand side). Consequently, we can choose $\delta$ so small that the exponent of the exponential in (7.41) is greater than in (7.32) and (7.36). This proves that the sign of the second derivative of the Poincaré map $P_{\varepsilon}$ in the case under consideration (the trajectory jumps downwards) is positive. The same arguments can be used to show that the sign is negative for $f\left(z_{n}, 0\right)>0$. This proves the lemma.

\section{ACKNOWLEDGEMENTS}

The author expresses his sincere gratitude to Yu. S. Ilyashenko for posing the problem, for his constant support and his interest in this work, and to V. Kleptsyn for numerous fruitful discussions and for his valuable comments on the text.

\section{REFERENCES}

[1] M. Diener, The canard unchained or how fast/slow dynamical systems bifurcate, Math. Intelligencer 6 (1984), no. 3, 38-49. MR748431 (85j:34120)

[2] F. Dumortier and R. Roussarie, Canard cycles and center manifolds, Mem. Amer. Math. Soc. 121 (1996), no. 577. MR 1327208 (96k:34113)

[3] J. Guckenheimer and Yu. Ilyashenko, The duck and the devil: canards on the staircase, Moscow Math. J. 1 (2001), no. 1, 27-47. MR1852932 (2002m:34083)

[4] M. Krupa and P. Szmolyan, Extending geometric singular perturbation theory to nonhyperbolic points - fold and canard points in two dimensions, SIAM J. Math. Anal. 33 (2001), no. 2, 286314 (electronic). MR1857972 (2002g:34117)

[5] I. Schurov, Ducks on the Torus: Existence and Uniqueness, J. Dynam. Control Systems 16 (2010), no. 2, 267-300; http://arxiv.org/abs/0910.1888. MR2646055

[6] N. Fenichel, Geometric singular perturbation theory for ordinary differential equations, J. Differential Equations 31 (1979), no. 1, 53-98. MR524817 (80m:58032)

[7] O. D. Anosova, On invariant manifolds in singularly perturbed systems, J. Dynam. Control Systems 5 (1999), no. 4, 501-507. MR.1722003 (2000h:37028) 
[8] O. D. Anosova, Invariant manifolds in singularly perturbed systems, Trudy Mat. Inst. Steklova 236 (2002), Differ. Uravn. i Din. Sist., 27-32; English transl., Proc. Steklov Inst. Math. 236 (2002), 19-24. MR 1931003 (2003g:34107)

[9] E. F. Mishchenko and N. Kh. Rozov, Differential equations with a small parameter, and relaxation oscillations, Nauka, Moscow, 1975; English transl., Plenum Press, New York, 1980. MR0508634 (58:22862) MR0750298(85j:34001)

Moscow State University

E-mail address: ilya@schurov.com 